Supplementary information for

\title{
Osmium complex-chromophore conjugates with both singlet-to-triplet absorption and long triplet lifetime through tuning of the heavy atom effect
}

\author{
Yoichi Sasaki, ${ }^{\dagger}$ Nobuhiro Yanai, ${ }^{*}, \uparrow, \uparrow$ Nobuo Kimizuka* ${ }^{*} \dagger$ \\ $\dagger$ Department of Applied Chemistry, Graduate School of Engineering, Center for Molecular Systems \\ (CMS), Kyushu University, 744 Moto-oka, Nishi-ku, Fukuoka 819-0395, Japan \\ II PRESTO, JST, Honcho 4-1-8, kawaguchi, Saitama 332-0012, Japan. \\ * To whom corresponding should be addressed. \\ yanai@mail.cstm.kyushu-u.ac.jp (N.Y.); n-kimi@mail.cstm.kyushu-u.ac.jp (N.K.)
}




\section{Materials}

All reagents and solvents were used as received without further purification otherwise noted. 2-Acetylpyridine was purchased from FUJIFILM Wako Pure Chemical Corporation. Potassium tert-butoxide, lithium bis(trifluoromethanesulfonyl)imide (LiTFSI), 4,4,5,5-tetramethyl-2-(3-perylenyl)1,3,2-dioxaborane, 4'-bromo-2,2':6',2"-terpyridine, 3-bromobenzaldehyde were purchased from TCI. Benzaldehyde and ammonium acetate were purchased from Kishida Chemical Co., Ltd. $\mathrm{OsCl}_{3} \cdot \mathrm{xH}_{2} \mathrm{O}$, $\mathrm{RuCl}_{3} \cdot \mathrm{xH}_{2} \mathrm{O}$, and tetrakis(triphenylphosphine)palladium( 0 ) were purchased from Sigma Aldrich. $\left[\operatorname{Ru}(\text { ptpy })_{2}\right]^{2+}, \quad\left[\operatorname{Ru}(\text { peptpy })_{2}\right]^{2+}, \quad\left[\mathrm{Os}(\text { ptpy })_{2}\right]^{2+}, \quad\left[\mathrm{Os}(\text { petpy })_{2}\right]^{2+}, \quad\left[\mathrm{Os}(\text { peptpy })_{2}\right]^{2+}$, $\left[\mathrm{Os}(m \text {-peptpy })_{2}\right]^{2+}$ were synthesized according to the methods described later. 3Phenylperylene (pPe), was synthesized according to the reported procedure. ${ }^{1}$ For spectroscopic measurements, dehydrated $N, N$-dimethylformamide (DMF) purchased from FUJIFILM Wako Pure Chemical Corporation was used.

\section{Characterizations}

${ }^{1} \mathrm{H}-\mathrm{NMR}(400 \mathrm{MHz})$ and ${ }^{13} \mathrm{C}-\mathrm{NMR}(101 \mathrm{MHz})$ spectra were measured on a JEOL JNM-ECZ400S using TMS as an internal standard. Mass spectroscopy analyses were conducted on a Bruker Autoflex III. Elemental analyses were conducted at the Elemental Analysis Center, Kyushu University. UV-Vis absorption spectra were recorded on a JASCO V-670 spectrophotometer. Absolute photoluminescence quantum yields were measured by using an absolute quantum yield measurement system C13534-01 (Hamamatsu Photonics). Luminescence spectra at room temperature and at $77 \mathrm{~K}$ were measured by using a JASCO FP-8700 NIR Spectrofluorometer. Time-resolved phosphorescence decays of Os(II) complexes were measured using a UNISOKU TSP-2000 system. Phosphorescence decay profiles of $\left[\mathrm{Os}(m \text {-peptpy })_{2}\right]^{2+}$ and $\left[\mathrm{Ru}(\text { peptpy })_{2}\right]^{2+}$ were measured by using a timecorrelated single photon counting lifetime spectroscopy system, HAMAMATSU Quantaurus-Tau C11567-01. Temperature-dependent PL decays were measured on a Deltaflex-NIR Model:KY1 system (HORIBA). Cyclic voltammetry measurements were performed by an ALS electrochemical analyzer Model 620D. 


\section{Density functional theory (DFT) calculations}

Density functional theory (DFT) and time-dependent DFT (TD-DFT) calculations were conducted with the B3LYP exchange-correlation functional as implemented in the Gaussian 16 software. ${ }^{2}$ As basis sets, SDD and 6-311G(d,p) were used for Os and other elements, respectively. All calculations were associated with the PCM model and $N, N$-dimethylformamide (DMF) was chosen as a solvent. Frequency analyses were also conducted to ensure the converged structures reached the potential energy minimums. Molecular orbitals were visualized by GaussView $6.0 .^{3}$

Synthetic Procedures ${ }^{1,4-6}$

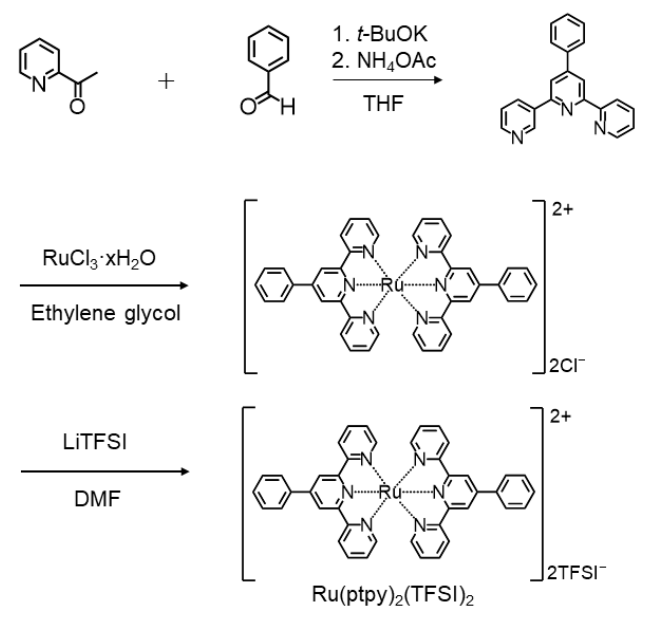

Scheme S-1. Synthetic scheme for Ru(ptpy $)_{2}(\mathrm{TFSI})_{2}$.

Synthesis of 4'-phenyl-2,2':6,2"-terpyridine (ptpy).

$1.4 \mathrm{~mL}(12 \mathrm{mmol})$ of 2-acetylpyridine and $2.0 \mathrm{~g}$ of potassium tert-butoxide were mixed into $50 \mathrm{~mL}$ of THF. $10 \mathrm{~mL}$ (ca. $5.7 \mathrm{mmol}$ ) of $5.7 \mathrm{v} / \mathrm{v} \%$ benzaldehyde THF solution was added, and the solution was stirred overnight at room temperature. 5.0 $\mathrm{g}$ of ammonium acetate was added and the solution was refluxed for 5.5 hours. The solution was concentrated under reduced pressure, and water was added until precipitates were formed. The solid was filtrated and purified by reprecipitation with acetone and water to afford pale orange powder of ptpy. (217 mg, 12\%) ${ }^{1} \mathrm{H}-\mathrm{NMR}$ $\left(400 \mathrm{MHz}\right.$, Chloroform- $d$ ): $\delta(\mathrm{ppm}) 8.75-8.73(\mathrm{~m}, 4 \mathrm{H}), 8.68\left(\mathrm{dt}, J_{1}=7.6 \mathrm{~Hz}, J_{2}=1.2\right.$ $\mathrm{Hz}, 2 \mathrm{H}), 7.93-7.87(\mathrm{~m}, 4 \mathrm{H}), 7.54-7.44(\mathrm{~m}, 3 \mathrm{H}), 7.36$ (ddd, $J_{1}=7.6 \mathrm{~Hz}, J_{2}=4.8 \mathrm{~Hz}$, $\left.J_{3}=1.2 \mathrm{~Hz}, 2 \mathrm{H}\right)$. 
Synthesis of Ru(ptpy $)_{2}(\mathrm{TFSI})_{2}$.

$31 \mathrm{mg}$ (ca. $0.15 \mathrm{mmol}$ ) of $\mathrm{RuCl}_{3} \cdot \mathrm{xH}_{2} \mathrm{O}$ and $93 \mathrm{mg}(0.30 \mathrm{mmol})$ of ptpy were dispersed into $2 \mathrm{~mL}$ of ethylene glycol and refluxed for 2 hours at $230{ }^{\circ} \mathrm{C}$ with microwave radiation (Biotage Initiator 2.5). Acetonitrile (ca. $25 \mathrm{~mL}$ ) and $432 \mathrm{mg}(1.5 \mathrm{mmol}$ ) of LiTFSI were added, then $50 \mathrm{~mL}$ of deionized water was added to give black precipitates. The precipitates were filtrated and washed with toluene. (50 mg, 26\%) ${ }^{1} \mathrm{H}-\mathrm{NMR}\left(400 \mathrm{MHz}, \mathrm{DMSO}-d_{6}\right): \delta(\mathrm{ppm}) 9.50(\mathrm{~s}, 4 \mathrm{H}), 9.13(\mathrm{~d}, J=8.0 \mathrm{~Hz}, 4 \mathrm{H}), 8.45$ $(\mathrm{d}, J=7.2 \mathrm{~Hz}, 4 \mathrm{H}), 8.08\left(\mathrm{td}, J_{1}=7.8 \mathrm{~Hz}, J_{2}=1.2 \mathrm{~Hz}, 4 \mathrm{H}\right), 7.79(\mathrm{t}, J=7.6 \mathrm{~Hz}, 4 \mathrm{H})$, $7.69(\mathrm{t}, J=7.2 \mathrm{~Hz}, 2 \mathrm{H}), 7.56(\mathrm{~d}, J=4.8 \mathrm{~Hz}, 4 \mathrm{H}), 7.29\left(\mathrm{ddd}, J_{1}=7.4 \mathrm{~Hz}, J_{2}=5.9 \mathrm{~Hz}\right.$, $\left.J_{3}=1.2 \mathrm{~Hz}, 4 \mathrm{H}\right),{ }^{13} \mathrm{C}-\mathrm{NMR}\left(101 \mathrm{MHz}, \mathrm{DMSO}-d_{6}\right): \delta(\mathrm{ppm}) 157.9,155.0,152.1$, $146.9,137.9,136.0,130.3,129.3,127.6,124.7,121.1,119.4$ (q, $\left.J_{\mathrm{FC}}=324 \mathrm{~Hz}, \mathrm{CF}_{3}\right)$, MS (MALDI): $\mathrm{m} / \mathrm{z}=718.91\left(\left[\mathrm{Ru}(\text { ptpy })_{2}-\mathrm{H}\right]^{+}\right)$, Elemental analysis: calculated for $\mathrm{C}_{46} \mathrm{H}_{30} \mathrm{~N}_{8} \mathrm{~F}_{12} \mathrm{O}_{8} \mathrm{RuS}_{4} \mathrm{C} 43.16 \mathrm{H} 2.36$ N 8.75, found C 43.18 H 2.35 N 8.78.

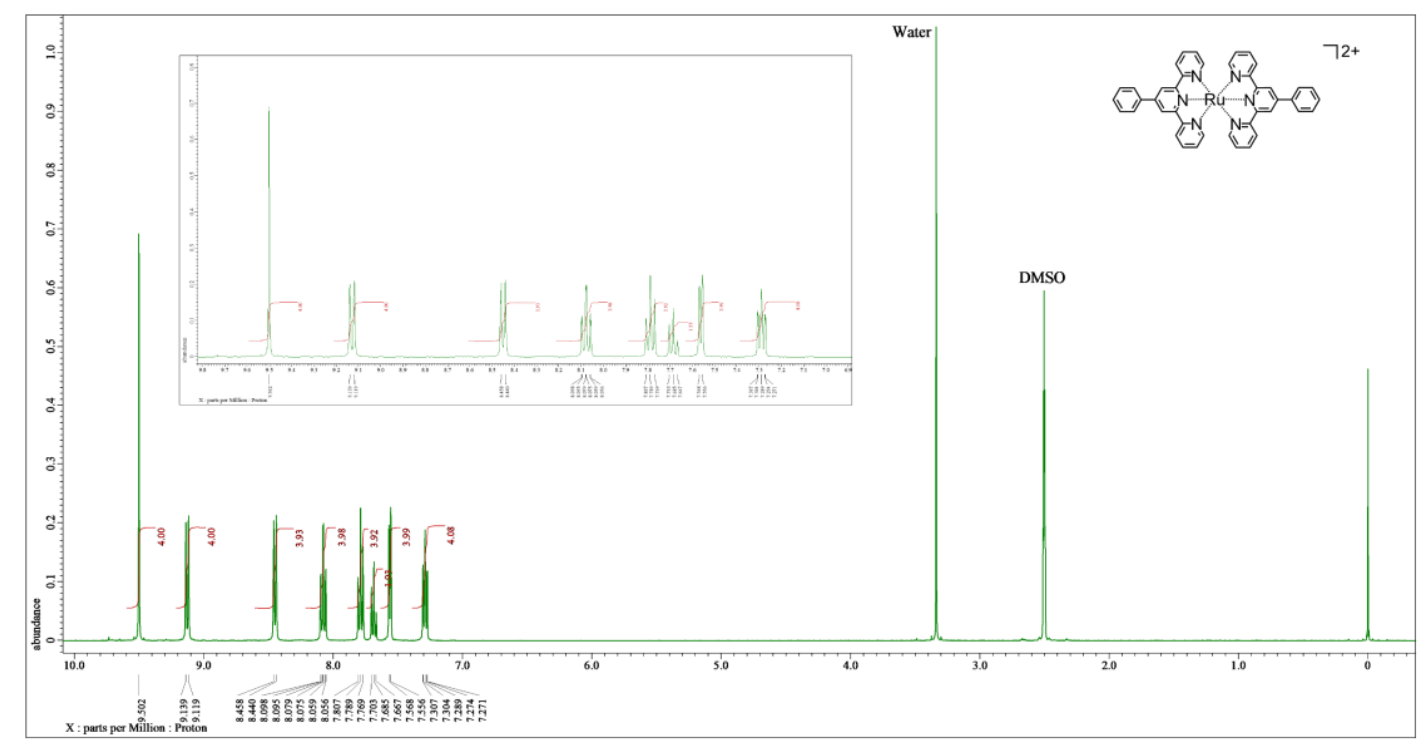

Figure S-1. ${ }^{1} \mathrm{H}-\mathrm{NMR}$ spectrum of Ru(ptpy) $)_{2}$ (TFSI) 2 (400 MHz, DMSO- $\left.d_{6}\right)$. 


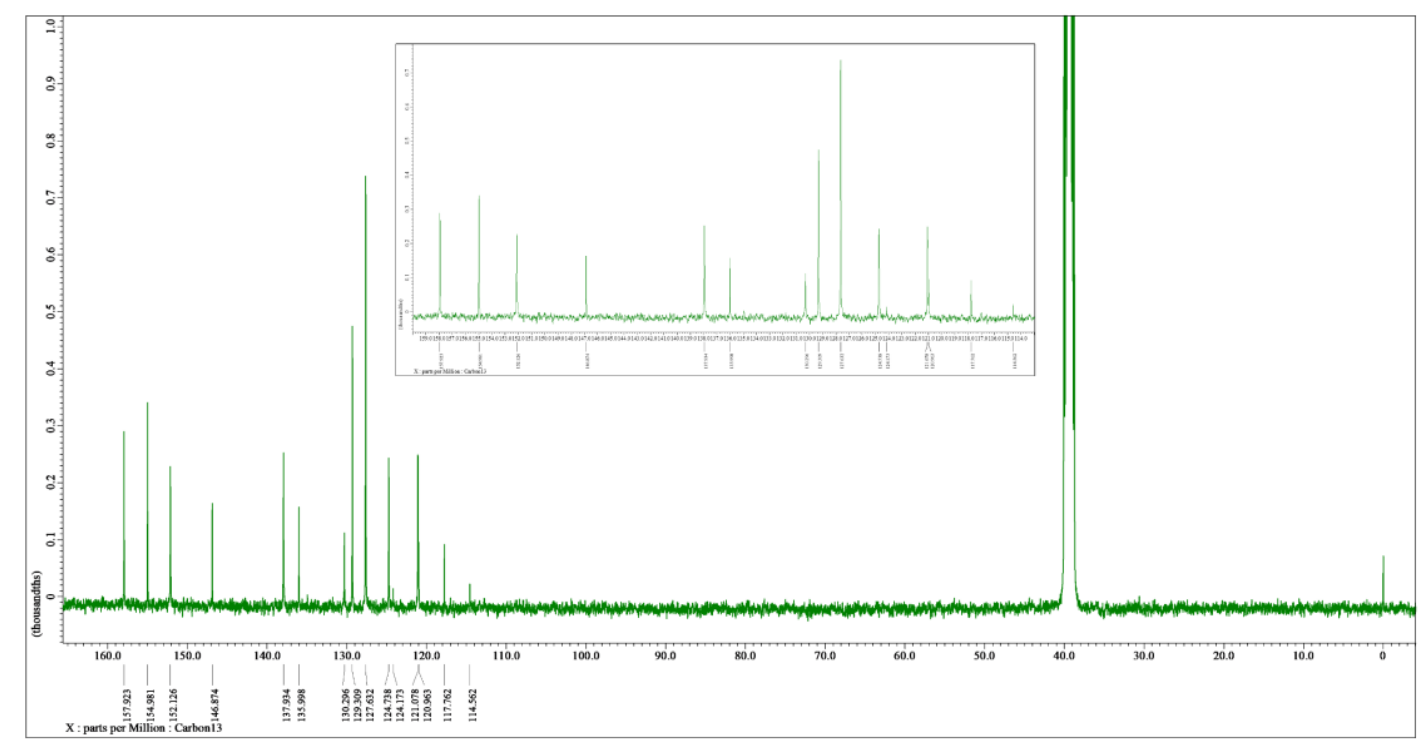

Figure S-2. ${ }^{13} \mathrm{C}-\mathrm{NMR}$ spectrum of Ru(ptpy $)_{2}(\mathrm{TFSI})_{2}\left(101 \mathrm{MHz}, \mathrm{DMSO}-d_{6}\right)$.

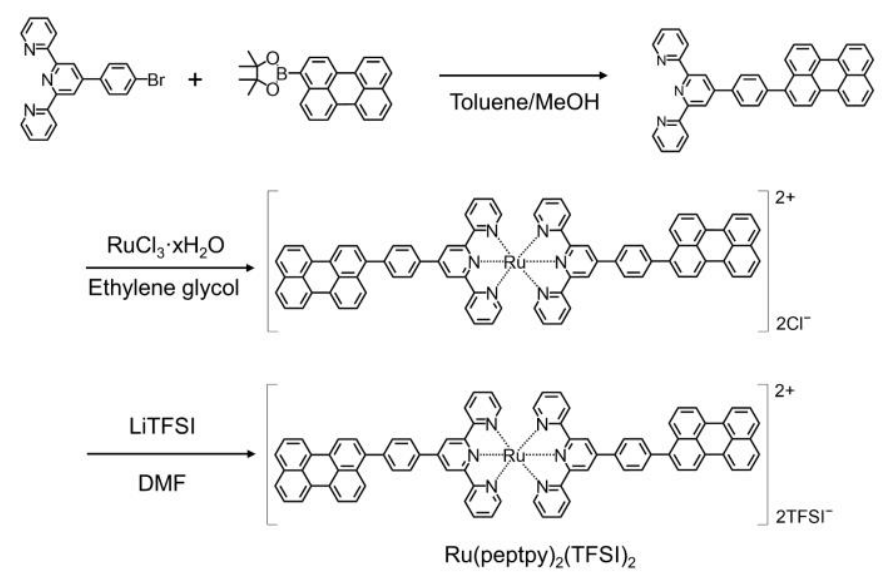

Scheme S-2. Synthetic scheme for Ru(peptpy) ${ }_{2}(\mathrm{TFSI})_{2}$.

Synthesis of 4'-(4-(perylen-3-yl) phenyl)-2,2':6',2"-terpyridine (peptpy)

$249 \mathrm{mg}(0.64 \mathrm{mmol})$ of 4'-(4-bromophenyl)-2,2':6',2"-terpyridine and $257 \mathrm{mg}$ (0.68 mmol) of 4,4,5,5-tetramethyl-2-(3-perylenyl)-1,3,2-dioxaborane, $553 \mathrm{mg}$ of potassium carbonate were dispersed into toluene/ $\mathrm{MeOH}$ (toluene: $\mathrm{MeOH}=60 \mathrm{~mL}$ : $90 \mathrm{~mL}) .46 \mathrm{mg}(0.04 \mathrm{mmol})$ of tetrakis(triphenylphosphine)palladium(0) was added and the solution was refluxed for 16 hours under $\mathrm{N}_{2}$ atmosphere. The solution was washed with $\mathrm{CHCl}_{3} / \mathrm{H}_{2} \mathrm{O}\left(\mathrm{CHCl}_{3}: \mathrm{H}_{2} \mathrm{O}=1: 1\right)$ and the organic phase was dried with 
sodium sulphate. After concentrating the solution, the compound was purified with column chromatography (column: $\mathrm{Al}_{2} \mathrm{O}_{3}$, solvents: $\mathrm{CHCl}_{3}$ and $\mathrm{MeOH}$ ). (275 mg, 77\%) ${ }^{1} \mathrm{H}-\mathrm{NMR}$ (400 MHz, Chloroform- $d$ ): $\delta(\mathrm{ppm}) 8.86(\mathrm{~s}, 2 \mathrm{H}), 8.76(\mathrm{~d}, J=4.6 \mathrm{~Hz}$, $2 \mathrm{H}), 8.71(\mathrm{~d}, J=8.0 \mathrm{~Hz}, 2 \mathrm{H}), 8.29-8.23(\mathrm{~m}, 4 \mathrm{H}), 8.08(\mathrm{~d}, J=8.0 \mathrm{~Hz}, 2 \mathrm{H}), 7.91(\mathrm{td}$, $\left.J_{1}=7.8 \mathrm{~Hz}, J_{2}=2.0 \mathrm{~Hz}, 2 \mathrm{H}\right), 7.83(\mathrm{~d}, J=8.0 \mathrm{~Hz}, 1 \mathrm{H}), 7.70(\mathrm{t}, J=8.4 \mathrm{~Hz}, 4 \mathrm{H}), 7.54-$ $7.46(\mathrm{~m}, 4 \mathrm{H}), 7.38\left(\mathrm{ddd}, J_{1}=7.6 \mathrm{~Hz}, J_{2}=4.8 \mathrm{~Hz}, J_{3}=1.2 \mathrm{~Hz}, 2 \mathrm{H}\right)$.

Synthesis of $\mathrm{Ru}(\text { peptpy })_{2}(\mathrm{TFSI})_{2}$.

$31 \mathrm{mg}$ (ca. $0.15 \mathrm{mmol}$ ) of $\mathrm{RuCl}_{3} \cdot \mathrm{xH}_{2} \mathrm{O}$ and $168 \mathrm{mg}(0.3 \mathrm{mmol})$ of peptpy were dispersed into $5 \mathrm{~mL}$ of ethylene glycol and refluxed for 2 hours at $230{ }^{\circ} \mathrm{C}$ with microwave radiation (Biotage Initiator 2.5). Water was added and the precipitates were filtrated. The solid was dissolved into $50 \mathrm{~mL}$ of DMF and mixed with $433 \mathrm{mg}$ ( $1.5 \mathrm{mmol}$ ) of LiTFSI at $60{ }^{\circ} \mathrm{C}$ for 3 hours. After cooling to room temperature, $50 \mathrm{~mL}$ of deionized water was added and the residue was filtrated. The precipitates were washed with toluene. The obtained solid was purified by reprecipitation with THF and toluene. The solid was again filtrated and washed with a large amount of toluene. (176 mg, 66\%) ${ }^{1} \mathrm{H}-\mathrm{NMR}$ (400 MHz, DMSO-d6): $\delta$ (ppm) $9.64(\mathrm{~s}, 4 \mathrm{H}), 9.20(\mathrm{~d}, J=$ $8.4 \mathrm{~Hz}, 4 \mathrm{H}), 8.66(\mathrm{~d}, J=8.4 \mathrm{~Hz}, 4 \mathrm{H}), 8.57-8.46(\mathrm{~m}, 8 \mathrm{H}), 8.12(\mathrm{t}, J=7.8 \mathrm{~Hz}, 4 \mathrm{H})$, $7.97(\mathrm{~d}, J=8.4 \mathrm{~Hz}, 4 \mathrm{H}), 7.87(\mathrm{t}, J=8.6 \mathrm{~Hz}, 6 \mathrm{H}), 7.70-7.60(\mathrm{~m}, 12 \mathrm{H}), 7.34(\mathrm{t}, J=6.6$ $\mathrm{Hz}, 4 \mathrm{H}),{ }^{13} \mathrm{C}-\mathrm{NMR}$ (101 MHz, DMSO- $\left.d_{6}\right): \delta$ (ppm) 158.0, 155.1, 152.2, 146.5, 141.8 , $138.3,138.0,135.3,134.2$, 132.1, 131.0, 130.6, 130.5, 130.4, 130.2, 128.4, 128.1, $127.9,127.7,127.3,127.0,125.2,124.8,121.1,121.0,120.9,120.5,119.4$ (q, $J_{\mathrm{FC}}=$ $\left.323 \mathrm{~Hz}, \mathrm{CF}_{3}\right), \mathrm{MS}(\mathrm{MALDI}): \mathrm{m} / \mathrm{z}=1219.11\left(\left[\mathrm{Ru}(\text { peptpy })_{2}-\mathrm{H}\right]^{+}\right)$, Elemental analysis: calculated for $\mathrm{C}_{86} \mathrm{H}_{50} \mathrm{~N}_{8} \mathrm{~F}_{12} \mathrm{O}_{8} \mathrm{RuS}_{4} \mathrm{C} 58.01 \mathrm{H} 2.83 \mathrm{~N} 6.29$, found C 57.73 H 2.79 N 6.15 . 


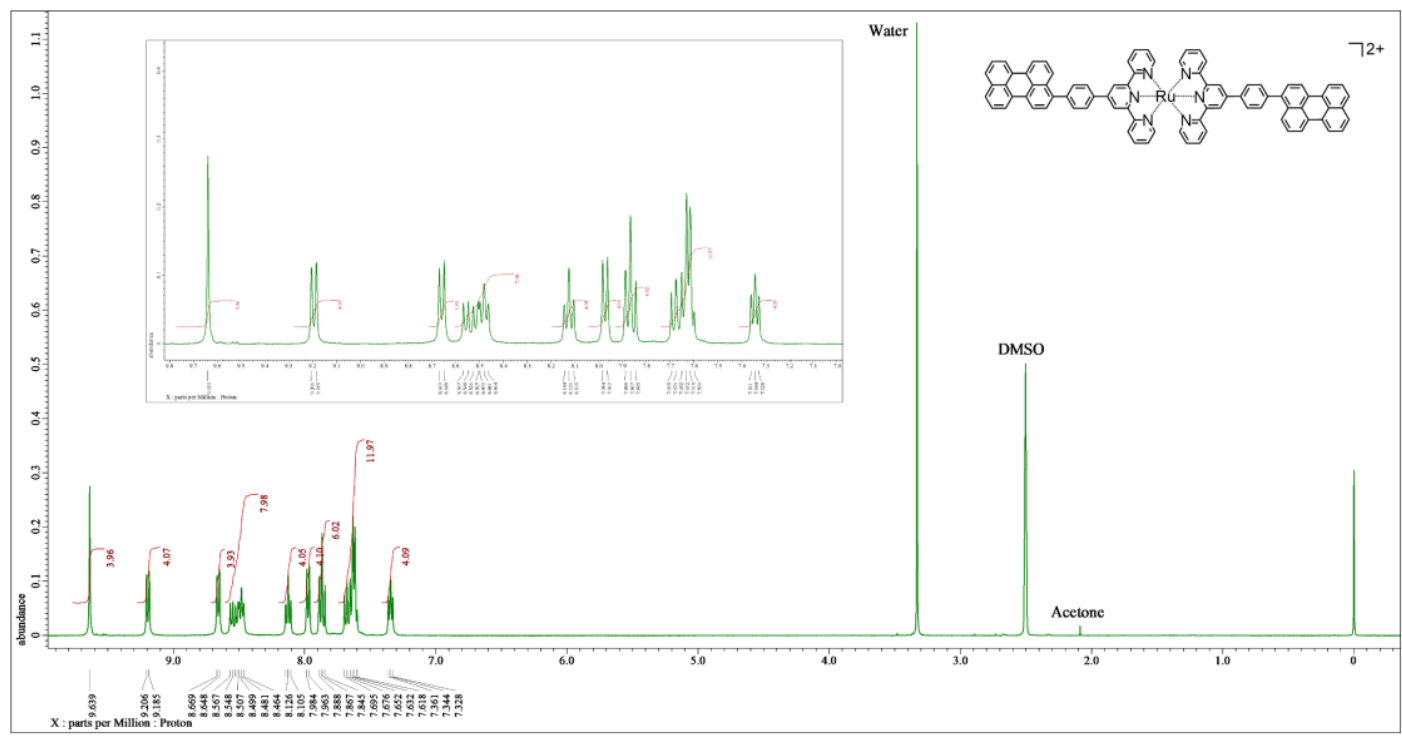

Figure S-3. ${ }^{1} \mathrm{H}-\mathrm{NMR}$ spectrum of Ru(peptpy) ${ }_{2}(\mathrm{TFSI})_{2}\left(400 \mathrm{MHz}, \mathrm{DMSO}-d_{6}\right)$.

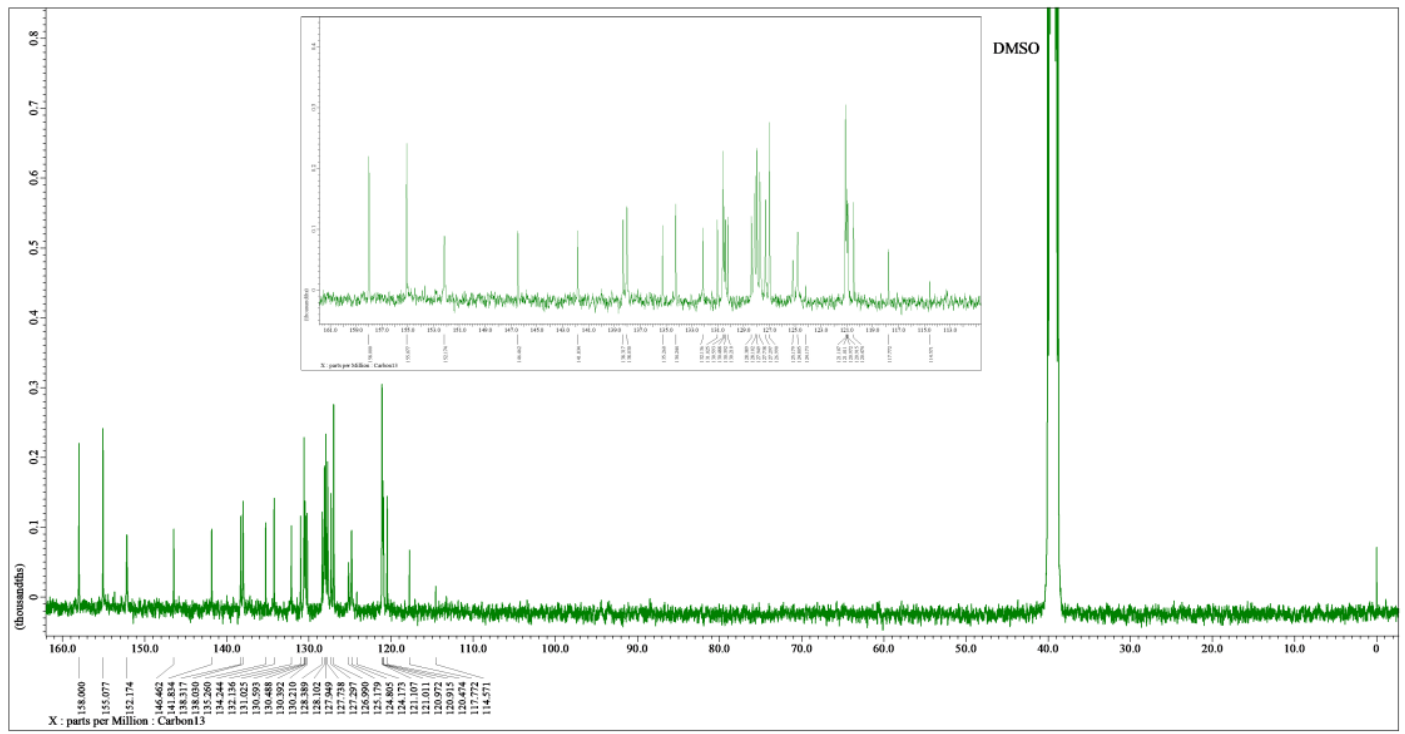

Figure S-4. ${ }^{13} \mathrm{C}-\mathrm{NMR}$ spectrum of Ru(peptpy) $)_{2}(\mathrm{TFSI})_{2}\left(101 \mathrm{MHz}, \mathrm{DMSO}-d_{6}\right)$. 


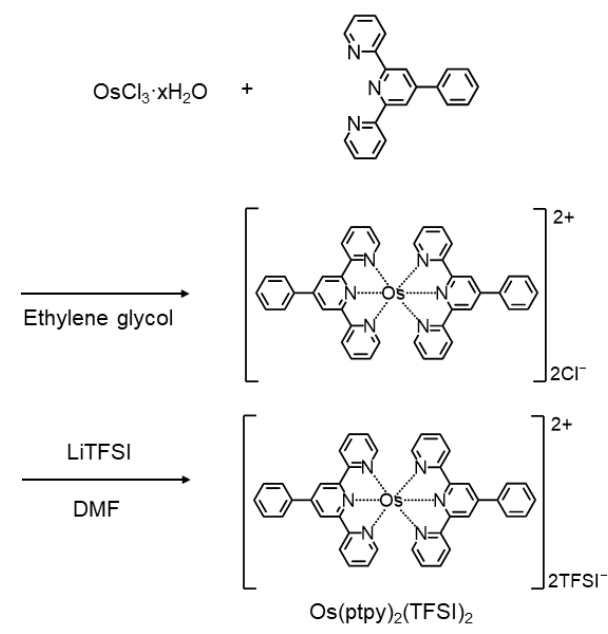

Scheme S-3. Synthetic scheme for Os(ptpy $)_{2}(\mathrm{TFSI})_{2}$.

Synthesis of Os(ptpy) 2 (TFSI) 2 .

$45 \mathrm{mg}$ (ca. $0.15 \mathrm{mmol}$ ) of $\mathrm{OsCl}_{3} \cdot \mathrm{xH}_{2} \mathrm{O}$ and $90 \mathrm{mg}(0.29 \mathrm{mmol})$ of ptpy were dispersed into $2 \mathrm{~mL}$ of ethylene glycol and refluxed for 2 hours at $230{ }^{\circ} \mathrm{C}$ with microwave radiation (Biotage Initiator 2.5). After the solution was mixed with acetone and 860 $\mathrm{mg}$ (3.0 mmol) of LiTFSI, water was added and stirred until black precipitates were produced. The precipitates were filtrated and washed with toluene. (156 $\mathrm{mg}, 79 \%$ ) ${ }^{1} \mathrm{H}-\mathrm{NMR}$ (400 MHz, DMSO- $d_{6}$ ): $\delta$ (ppm) 9.52 (s, 4H), 9.12 (d, $J=8.0 \mathrm{~Hz}, 4 \mathrm{H}$ ), 8.41 $(\mathrm{d}, J=7.2 \mathrm{~Hz}, 4 \mathrm{H}), 7.94\left(\mathrm{td}, J_{1}=7.9 \mathrm{~Hz}, J_{2}=1.1 \mathrm{~Hz}, 4 \mathrm{H}\right), 7.78(\mathrm{t}, J=7.8 \mathrm{~Hz}, 4 \mathrm{H})$, $7.60(\mathrm{t}, J=7.4 \mathrm{~Hz}, 2 \mathrm{H}), 7.44(\mathrm{~d}, J=5.2 \mathrm{~Hz}, 4 \mathrm{H}), 7.22\left(\mathrm{ddd}, J_{1}=7.3 \mathrm{~Hz}, J_{2}=5.9 \mathrm{~Hz}\right.$, $\left.J_{3}=0.9 \mathrm{~Hz}, 4 \mathrm{H}\right),{ }^{13} \mathrm{C}-\mathrm{NMR}\left(101 \mathrm{MHz}, \mathrm{DMSO}-d_{6}\right): \delta(\mathrm{ppm}) 159.7,154.6,152.1$, $146.1,137.7,135.3,130.3,129.1,127.9,127.9,124.9,119.7,119.4$ (q, $J_{\mathrm{FC}}=323 \mathrm{~Hz}$, $\left.\mathrm{CF}_{3}\right), \mathrm{MS}$ (MALDI): $\mathrm{m} / \mathrm{z}=808.83\left(\left[\mathrm{Os}(\mathrm{ptpy})_{2}-\mathrm{H}\right]^{+}\right)$, Elemental analysis: calculated for $\mathrm{C}_{46} \mathrm{H}_{30} \mathrm{~N}_{8} \mathrm{~F}_{12} \mathrm{O}_{8} \mathrm{OsS}_{4} \mathrm{C} 40.35 \mathrm{H} 2.21 \mathrm{~N} 8.18$, found $\mathrm{C} 40.45 \mathrm{H} 2.15 \mathrm{~N}$ 8.34. 


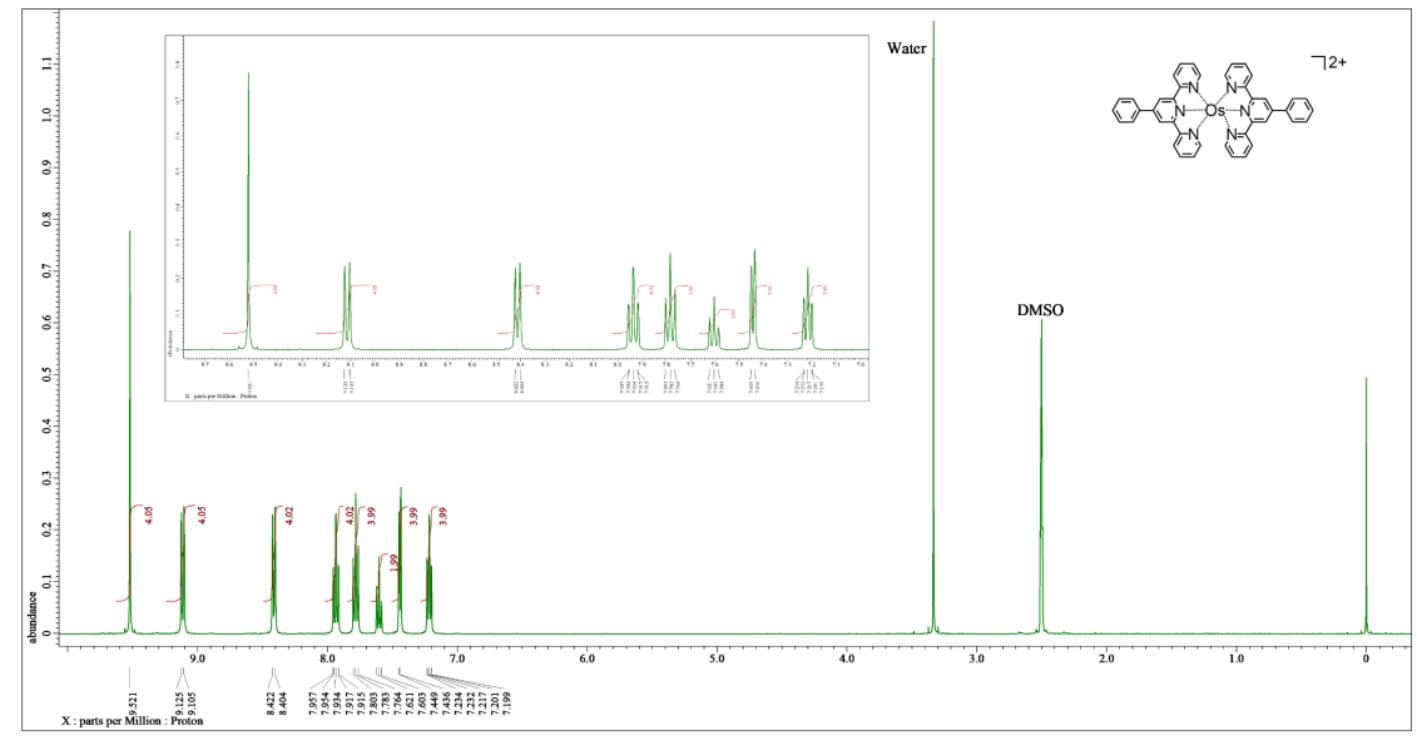

Figure S-5. ${ }^{1} \mathrm{H}-\mathrm{NMR}$ spectrum of Os(ptpy) $)_{2}$ (TFSI) $)_{2}\left(400 \mathrm{MHz}, \mathrm{DMSO}-d_{6}\right)$.

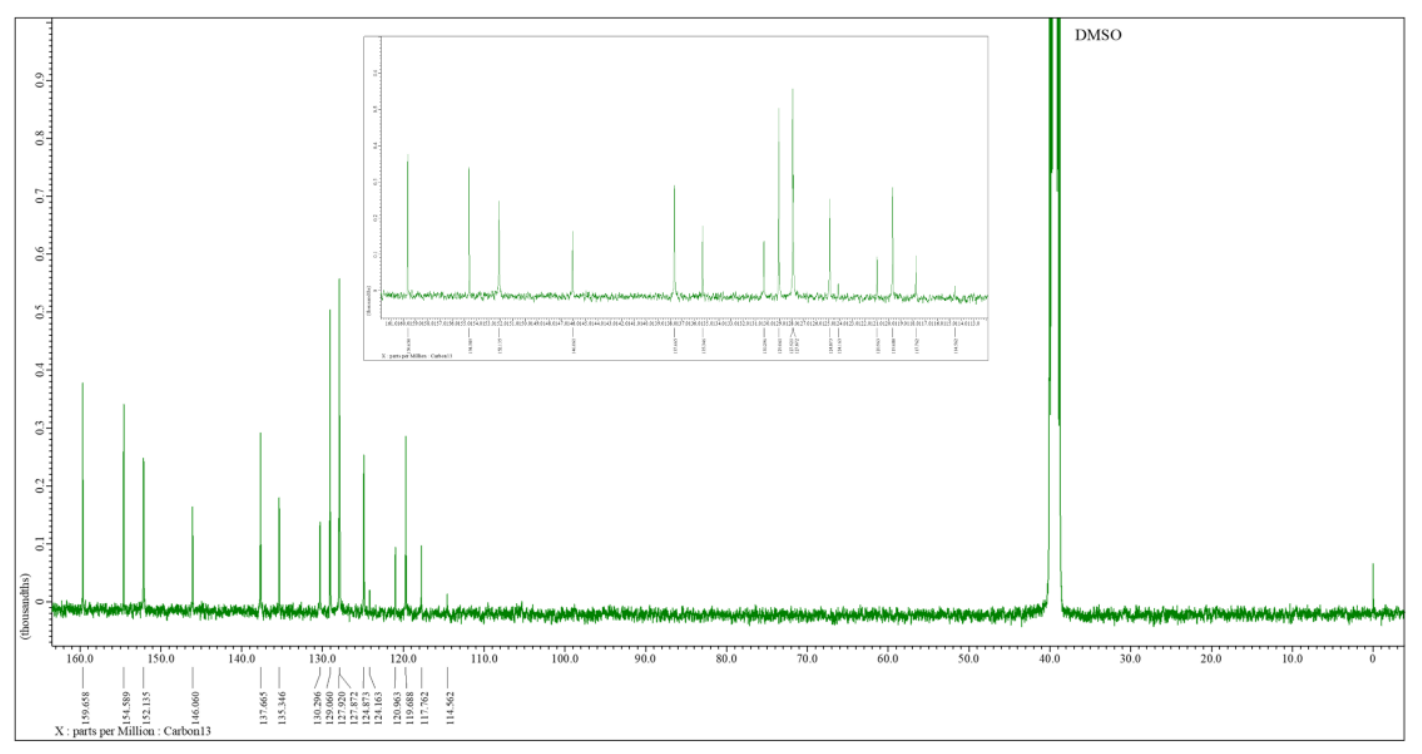

Figure S-6. ${ }^{13} \mathrm{C}-\mathrm{NMR}$ spectrum of Os(ptpy) $)_{2}(\mathrm{TFSI})_{2}\left(101 \mathrm{MHz}, \mathrm{DMSO}-d_{6}\right)$. 


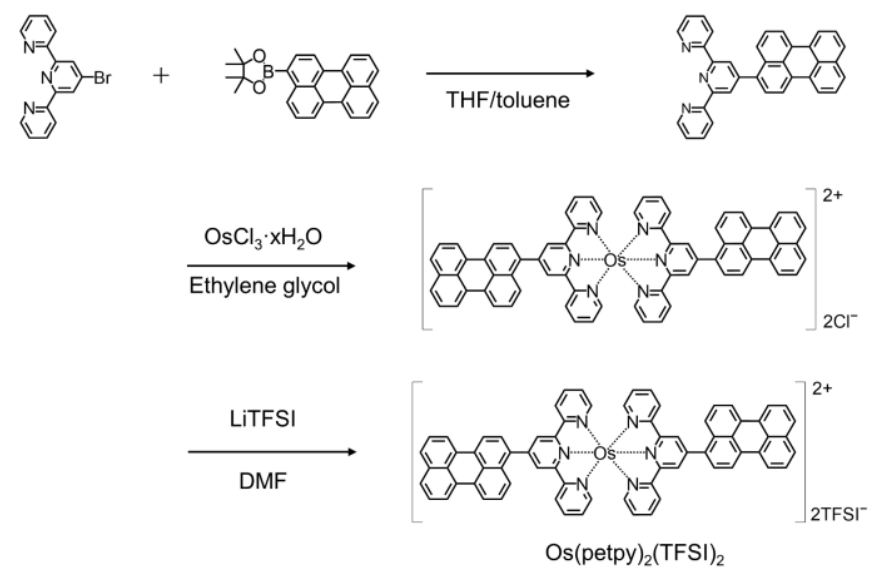

Scheme S-4. Synthetic scheme for Os(petpy $)_{2}(\mathrm{TFSI})_{2}$.

Synthesis of 4'-(perylen-3-yl)-2,2':6',2"-terpyridine (petpy).

$454 \mathrm{mg}(1.2 \mathrm{mmol})$ of 4,4,5,5-tetramethyl-2-(3-perylenyl)-1,3,2-dioxaborane and $312 \mathrm{mg}$ (1.0 mmol) of 4'-bromo-2,2':6',2"-terpyridine were dissolved in THF/toluene (THF: toluene $=10 \mathrm{~mL}: 30 \mathrm{~mL}$ ). $2 \mathrm{~mL}$ of aqueous solution of $2 \mathrm{M} \mathrm{K}_{2} \mathrm{CO}_{3}$ and 57.7 $\mathrm{mg}(0.05 \mathrm{mmol})$ of tetrakis(triphenylphosphine)palladium $(0)$ were added and the solution was refluxed for 12 hours under $\mathrm{N}_{2}$. The solution was washed with toluene/ $\mathrm{H}_{2} \mathrm{O}$ (toluene: $\mathrm{H}_{2} \mathrm{O}=1: 1$ ) and the precipitates were dissolved in hot toluene solution and filtrated. The solution was condensed and cooled to room temperature. Then the objective compound was purified by reprecipitation with toluene and hexane. (89.4 mg, 18\%) ${ }^{1} \mathrm{H}-\mathrm{NMR}$ (400 MHz, Chloroform- $d$ ): $\delta$ (ppm) 8.73-8.69 (m, $4 \mathrm{H}), 8.66(\mathrm{~s}, 2 \mathrm{H}), 8.28-8.23(\mathrm{~m}, 4 \mathrm{H}), 7.91\left(\mathrm{td}, J_{1}=7.8 \mathrm{~Hz}, J_{2}=2.0 \mathrm{~Hz}, 2 \mathrm{H}\right), 7.82(\mathrm{~d}$, $J=8.0 \mathrm{~Hz}, 1 \mathrm{H}), 7.72\left(\mathrm{dd}, J_{1}=8.0 \mathrm{~Hz}, J_{2}=3.5 \mathrm{~Hz}, 2 \mathrm{H}\right), 7.59(\mathrm{~d}, J=7.2 \mathrm{~Hz}, 1 \mathrm{H})$, 7.54-7.45 (m, 3H), $7.36\left(\mathrm{ddd}, J_{1}=7.6 \mathrm{~Hz}, J_{2}=4.8 \mathrm{~Hz}, J_{3}=1.2 \mathrm{~Hz}, 2 \mathrm{H}\right)$.

Synthesis of Os(petpy) 2 (TFSI) 2 .

Osmium(III) chloride hydrate (27 mg, ca. $0.09 \mathrm{mmol})$ and petpy (72 mg, $0.15 \mathrm{mmol}$ ) were refluxed in $5 \mathrm{~mL}$ of ethylene glycol for 2 hours at $230{ }^{\circ} \mathrm{C}$ using microwave radiation (Biotage Initiator 2.5). After cooling to room temperature, deionized water was added. Then $1.5 \mathrm{M}$ aqueous solution of LiTFSI ( $1 \mathrm{~mL}, 1.5 \mathrm{mmol})$ was added, giving dark purple precipitates. The product was filtrated and dispersed into hot toluene at $100^{\circ} \mathrm{C}$. After the filtration and washing with hexane and $\mathrm{CHCl}_{3}$, the residue was dissolved into THF. The solution was filtrated and condensed. Finally, 
recrystallization with hexane/THF solution gave a purified product of Os(petpy) $)_{2}(\mathrm{TFSI})_{2} .(56 \mathrm{mg}, 43 \%){ }^{1} \mathrm{H}-\mathrm{NMR}\left(400 \mathrm{MHz}, \mathrm{DMSO}-d_{6}\right): \delta(\mathrm{ppm}) 9.40$ (s, $4 \mathrm{H}), 9.01$ (d, $J=8.4 \mathrm{~Hz}, 4 \mathrm{H}), 8.76$ (d, $J=7.6 \mathrm{~Hz}, 2 \mathrm{H}), 8.61$ (t, $J=8.2 \mathrm{~Hz}, 4 \mathrm{H}), 8.53$ (d, $J=8.0 \mathrm{~Hz}, 2 \mathrm{H}), 8.37$ (d, $J=8.8 \mathrm{~Hz}, 2 \mathrm{H}), 8.00(\mathrm{~d}, J=7.6 \mathrm{~Hz}, 2 \mathrm{H}), 7.94-7.90(\mathrm{~m}$, $8 \mathrm{H}), 7.77(\mathrm{t}, J=8.0 \mathrm{~Hz}, 2 \mathrm{H}), 7.70-7.63(\mathrm{~m}, 8 \mathrm{H}), 7.31(\mathrm{t}, J=6.8 \mathrm{~Hz}, 4 \mathrm{H}),{ }^{13} \mathrm{C}-\mathrm{NMR}$ (101 MHz, DMSO- $\left.d_{6}\right): \delta(\mathrm{ppm}) 159.7,154.4,152.4,150.1,146.7,137.8,135.4$, $134.2,132.5,131.9,131.1,130.2,129.9,128.7,128.3,127.9,127.8,127.0,127.0$, 125.9, 124.8, 123.6, 121.6, 121.4, 121.3, 120.3, $119.4\left(\mathrm{q}, J_{\mathrm{FC}}=323 \mathrm{~Hz}, \mathrm{CF}_{3}\right), \mathrm{MS}$ (MALDI): $\mathrm{m} / \mathrm{z}=1155.31\left(\left[\mathrm{Os}(\text { petpy })_{2}-\mathrm{H}\right]^{+}\right)$Elemental analysis: calculated for $\mathrm{C}_{74} \mathrm{H}_{42} \mathrm{~N}_{8} \mathrm{~F}_{12} \mathrm{O}_{8} \mathrm{OsS}_{4} \mathrm{C} 51.75 \mathrm{H} 2.46 \mathrm{~N}$ 6.52, Found C $51.74 \mathrm{H} 2.53$ N 6.28.

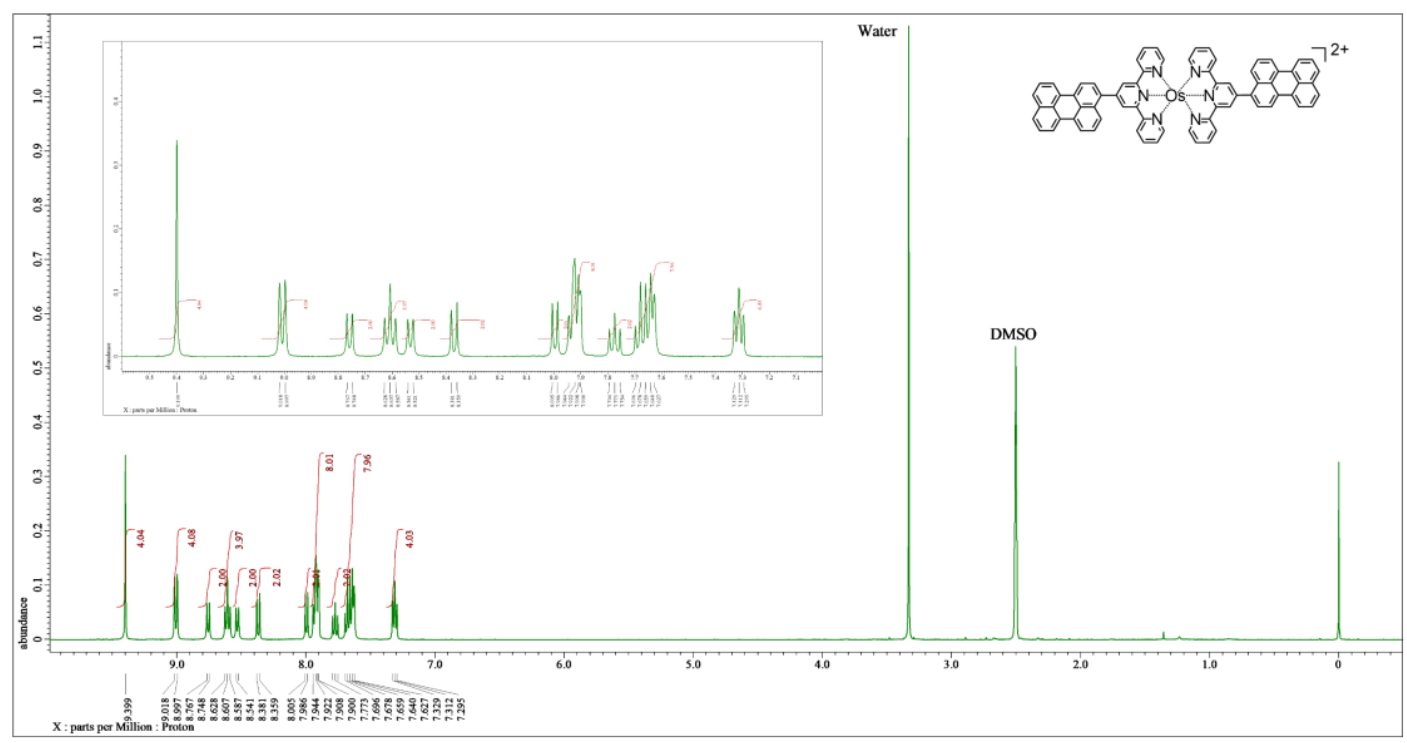

Figure S-7. ${ }^{1} \mathrm{H}-\mathrm{NMR}$ spectrum of Os(petpy $)_{2}(\mathrm{TFSI})_{2}\left(400 \mathrm{MHz}\right.$, DMSO- $\left.d_{6}\right)$. 


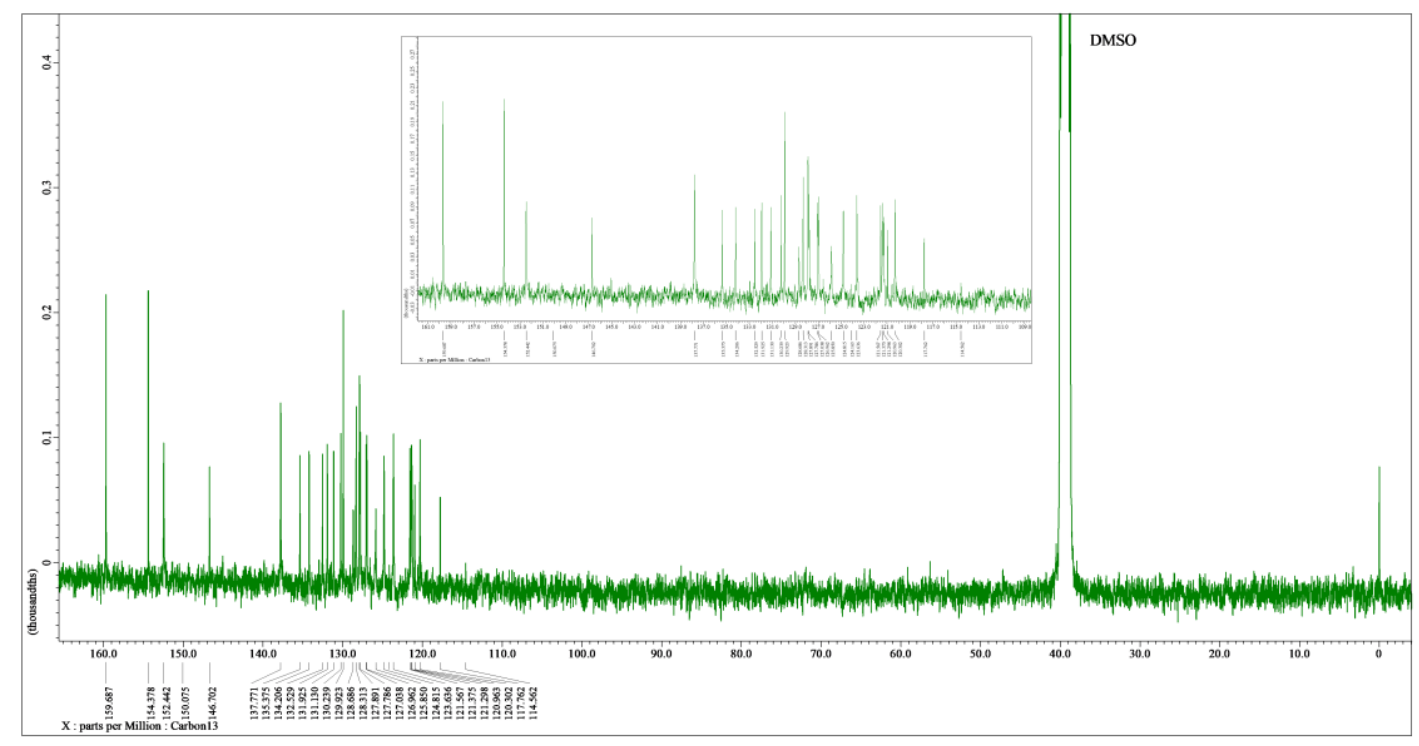

Figure S-8. ${ }^{13} \mathrm{C}-\mathrm{NMR}$ spectrum of Os(petpy $)_{2}(\mathrm{TFSI})_{2}\left(101 \mathrm{MHz}, \mathrm{DMSO}-d_{6}\right)$.

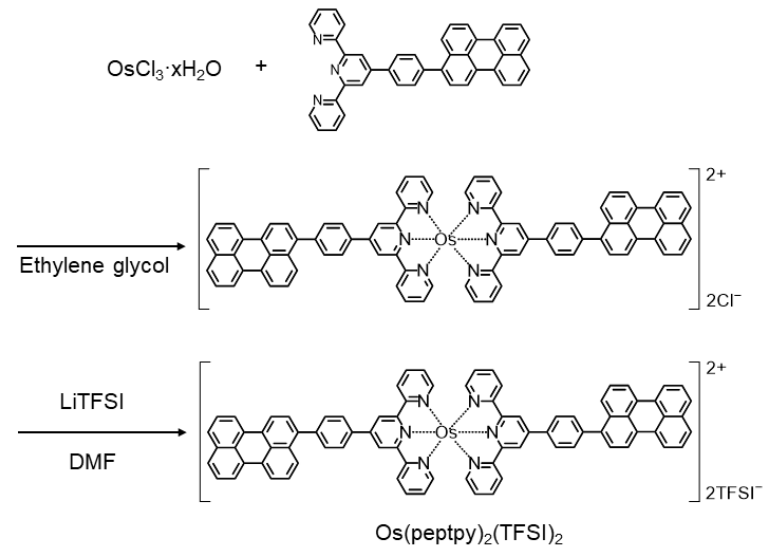

Scheme S-5. Synthetic scheme for Os(peptpy $)_{2}(\mathrm{TFSI})_{2}$.

Synthesis of Os(peptpy $)_{2}(\mathrm{TFSI})_{2}$

$44 \mathrm{mg}$ (ca. $0.15 \mathrm{mmol})$ of $\mathrm{OsCl}_{3} \cdot \mathrm{xH}_{2} \mathrm{O}$ and $168 \mathrm{mg}(0.30 \mathrm{mmol})$ of peptpy were dispersed into $5 \mathrm{~mL}$ of ethylene glycol and refluxed for 2 hours at $230{ }^{\circ} \mathrm{C}$ with microwave radiation (Biotage Initiator 2.5). After cooling to room temperature, 433 $\mathrm{mg}(1.5 \mathrm{mmol})$ of LiTFSI and $30 \mathrm{~mL}$ of DMF were added. The solution was stirred and water was added until black precipitates were produced. The precipitates were filtrated and washed with water. The solid was dispersed into hot toluene and 
collected by filtration. The solid was washed with toluene to afford the solid of Os(peptpy) $)_{2}$ (TFSI)2. (129 mg, 46\%) ${ }^{1} \mathrm{H}-\mathrm{NMR}$ (400 MHz, DMSO- $\left.d_{6}\right): \delta$ (ppm) 9.66 (s, 4H), 9.18 (d, $J=8.4 \mathrm{~Hz}, 4 \mathrm{H}), 8.62$ (d, $J=8.0 \mathrm{~Hz}, 4 \mathrm{H}), 8.57-8.47$ (m, 8H), 8.01$7.96(\mathrm{~m}, 8 \mathrm{H}), 7.87$ (t, $J=7.6 \mathrm{~Hz}, 6 \mathrm{H}), 7.69-7.60(\mathrm{~m}, 8 \mathrm{H}), 7.50(\mathrm{~d}, J=6.0 \mathrm{~Hz}, 4 \mathrm{H})$, $7.27(\mathrm{t}, J=6.4 \mathrm{~Hz}, 4 \mathrm{H}),{ }^{13} \mathrm{C}-\mathrm{NMR}\left(101 \mathrm{MHz}, \mathrm{DMSO}-d_{6}\right): \delta(\mathrm{ppm}) 159.7,154.7$, $152.2,145.6,141.9,138.3,137.8,134.6,134.2$, 132.2, 131.0, 130.5, 130.4, 130.3, $130.2,128.4,128.2,127.9,127.8,127.3,127.0,125.2$, 124.9, 121.1, 120.9, 120.5, 119.7, $119.4\left(\mathrm{q}, \quad J_{\mathrm{FC}}=323 \mathrm{~Hz}, \mathrm{CF}_{3}\right), \quad \mathrm{MS}(\mathrm{MALDI}): \mathrm{m} / \mathrm{z}=1309.90$ $\left(\left[\mathrm{Os}(\text { peptpy })_{2}-\mathrm{H}\right]^{+}\right), \quad 1589.92 \quad\left[\left(\mathrm{Os}(\text { peptpy })_{2}(\mathrm{TFSI})\right]^{+}\right), \quad$ Elemental analysis: calculated for $\mathrm{C}_{86} \mathrm{H}_{50} \mathrm{~N}_{8} \mathrm{~F}_{12} \mathrm{O}_{8} \mathrm{OsS}_{4}: \mathrm{C} 55.24 \mathrm{H} 2.70 \mathrm{~N} 5.99$, found C $55.15 \mathrm{H} 2.71 \mathrm{~N}$ 6.02 .

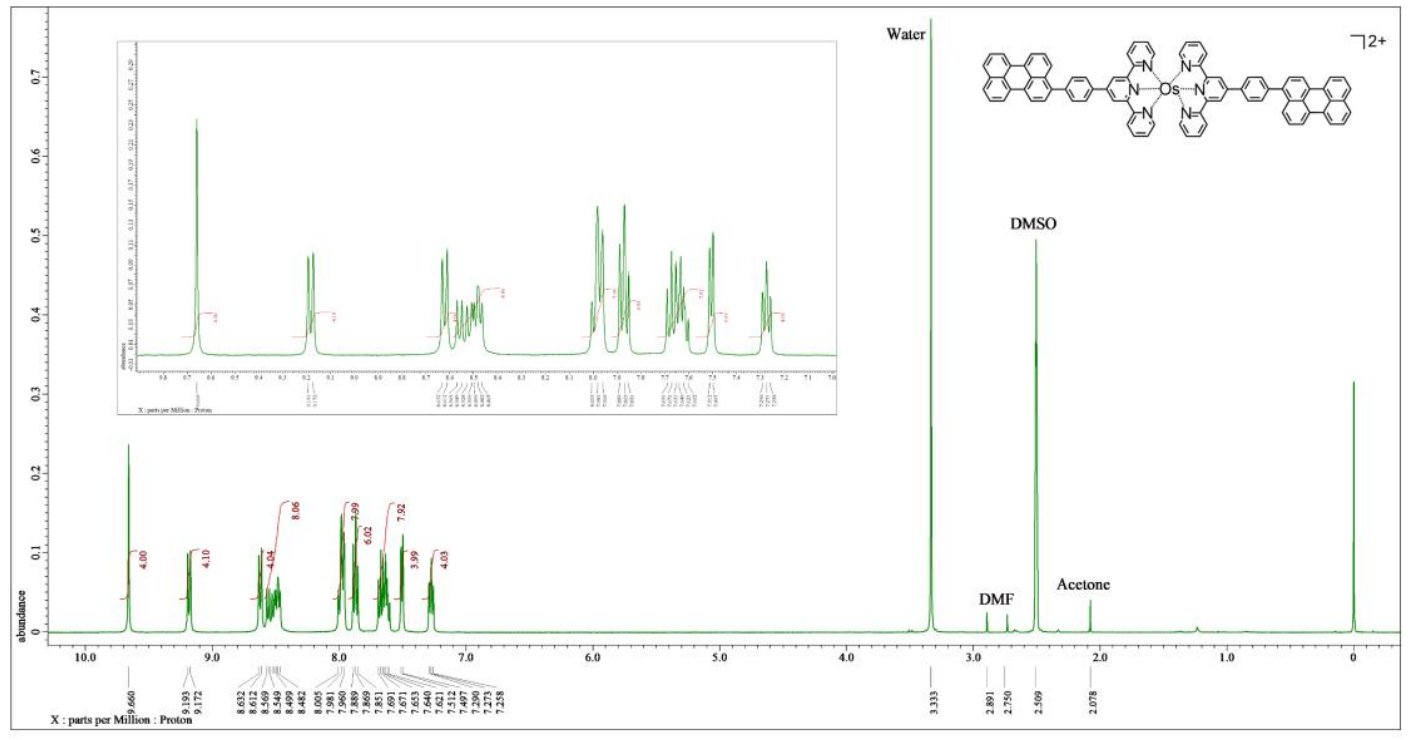

Figure S-9. ${ }^{1} \mathrm{H}-\mathrm{NMR}$ spectrum of Os(peptpy $)_{2}(\mathrm{TFSI})_{2}\left(400 \mathrm{MHz}, \mathrm{DMSO}-d_{6}\right)$. 


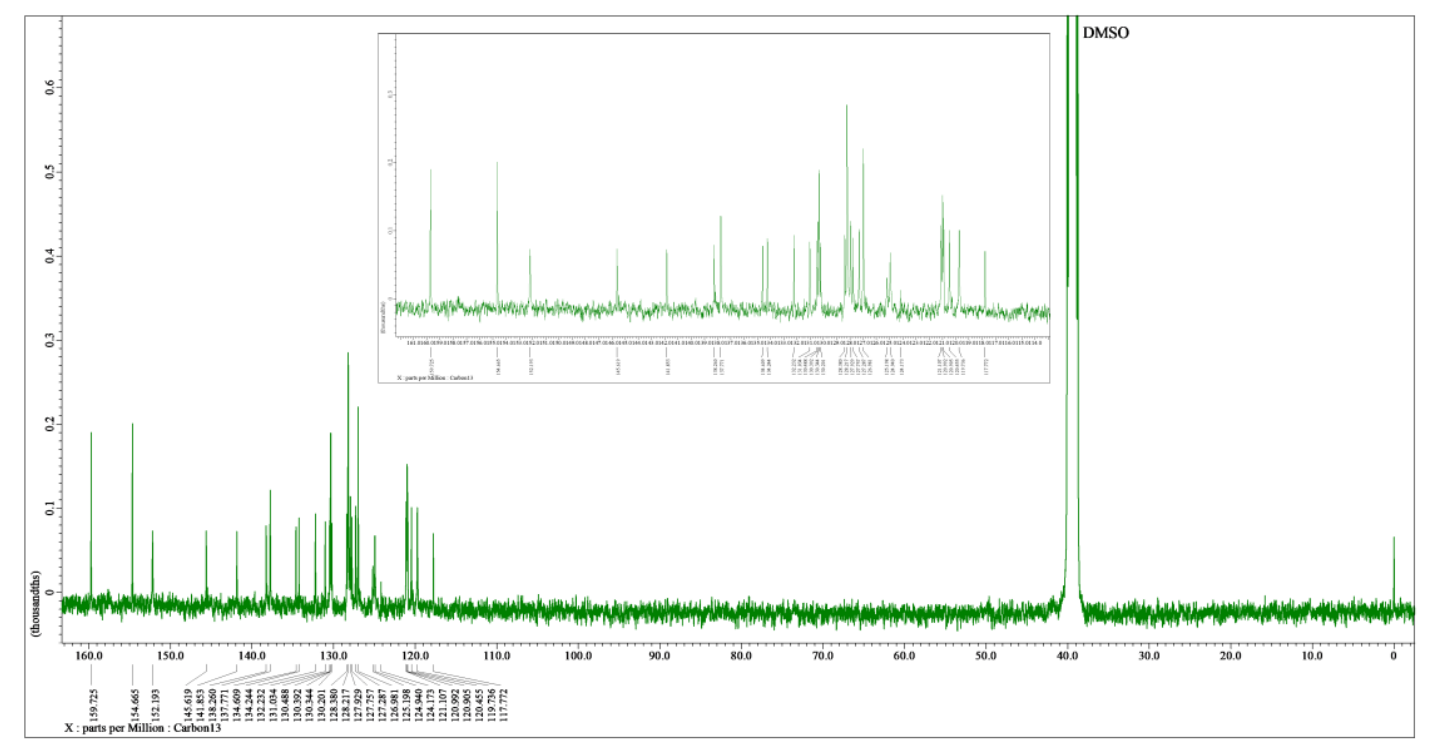

Figure S-10. ${ }^{13} \mathrm{C}-\mathrm{NMR}$ spectrum of Os(peptpy) $)_{2}(\mathrm{TFSI})_{2}\left(101 \mathrm{MHz}, \mathrm{DMSO}-d_{6}\right)$. A peak expected at $114.6 \mathrm{ppm}$ for $\mathrm{CF}_{3}$ in $\mathrm{TFSI}^{-}$was not observed with sufficient resolution, but peaks at 124.2, 121.0, 117.8 were analyzed as a quartet.

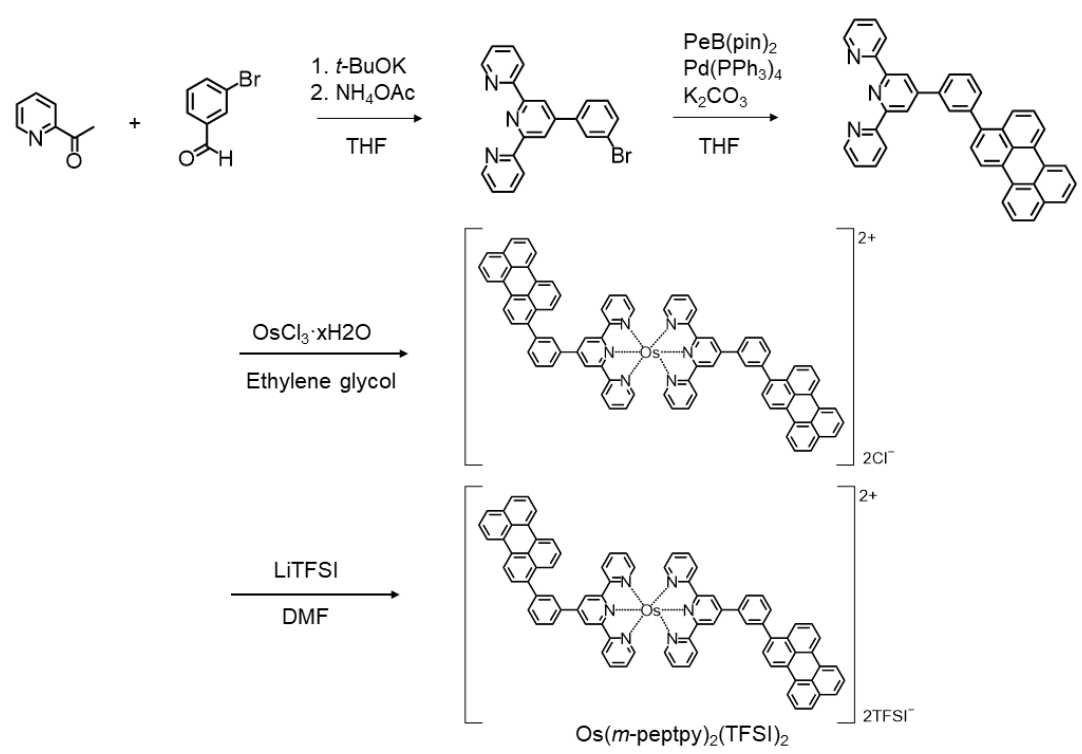

Scheme S-6. Synthetic scheme for Os(m-peptpy $)_{2}$ (TFSI $)_{2}$.

Synthesis of 4'-(3-bromophenyl)-2,2':6',2"-terpyridine ( $m$-bptpy).

$2.0 \mathrm{~mL}(17.8 \mathrm{mmol})$ of 2-acetylpyridine and $3.0 \mathrm{~g}$ of potassium tert-butoxide were mixed into $75 \mathrm{~mL}$ of THF. $15 \mathrm{~mL}$ (ca. $6.7 \mathrm{mmol}$ ) of $5.3 \mathrm{v} / \mathrm{v} \% 3$-bromobenzaldehyde THF solution was added, and the solution was stirred at room temperature. $7.5 \mathrm{~g}$ of ammonium acetate was added and the solution was refluxed for 7.5 hours. The 
solution was concentrated under reduced pressure, and water was added until precipitates were formed. The solid was filtrated and purified by reprecipitation with $\mathrm{MeOH}$ and $\mathrm{CHCl}_{3}$ to afford pale yellow powder of $m$-bptpy. $(1.49 \mathrm{~g}, 57 \%){ }^{1} \mathrm{H}-\mathrm{NMR}$ (400 MHz, Chloroform- $d$ ) $\delta(\mathrm{ppm}) 8.74(\mathrm{~d}, J=4.8 \mathrm{~Hz}, 2 \mathrm{H}), 8.70(\mathrm{~s}, 2 \mathrm{H}), 8.68(\mathrm{~d}, J$ $=8.4 \mathrm{~Hz}, 2 \mathrm{H}), 8.05(\mathrm{t}, J=1.8 \mathrm{~Hz}, 1 \mathrm{H}), 7.90\left(\mathrm{td}, J_{1}=7.7 \mathrm{~Hz}, J_{2}=1.8 \mathrm{~Hz}, 2 \mathrm{H}\right), 7.83$ $(\mathrm{d}, J=7.6 \mathrm{~Hz}, 1 \mathrm{H}), 7.59$ (d, $J=8.4 \mathrm{~Hz}, 1 \mathrm{H}), 7.41-7.36(\mathrm{~m}, 3 \mathrm{H})$.

Synthesis of 4'-(3-(perylen-3-yl)phenyl)-2,2':6',2"-terpyridine ( $m$-peptpy).

$249 \mathrm{mg}(0.64 \mathrm{mmol})$ of $m$-bptpy and $257 \mathrm{mg}(0.68 \mathrm{mmol})$ of 4,4,5,5-tetramethyl-2(3-perylenyl)-1,3,2-dioxaborane (PeB(pin)2), $556 \mathrm{mg}$ of potassium carbonate were dispersed into toluene/MeOH (toluene: $\mathrm{MeOH}=60 \mathrm{~mL}: 90 \mathrm{~mL}) .47 \mathrm{mg}(0.04 \mathrm{mmol})$ of tetrakis(triphenylphosphine)palladium(0) was added and the solution was refluxed for 12 hours under $\mathrm{N}_{2}$ atomosphere. The solution was washed with $\mathrm{CHCl}_{3} / \mathrm{H}_{2} \mathrm{O}$ $\left(\mathrm{CHCl}_{3}: \mathrm{H}_{2} \mathrm{O}=1: 1\right)$ and the organic phase was dried with sodium sulphate. After concentrating the solution, the compound was purified with column chromatography (column: $\mathrm{Al}_{2} \mathrm{O}_{3}$, solvent: $\mathrm{CHCl}_{3}$ ). (208 mg, 58\%) ${ }^{1} \mathrm{H}-\mathrm{NMR}$ (400 MHz, Chloroformd): $\delta(\mathrm{ppm}) 8.80(\mathrm{~s}, 2 \mathrm{H}), 8.71\left(\mathrm{dt}, J_{1}=4.8 \mathrm{~Hz}, J_{2}=0.9 \mathrm{~Hz}, 2 \mathrm{H}\right), 8.68(\mathrm{~d}, J=7.6 \mathrm{~Hz}$, 2H), 8.28-8.21 (m, 4H), 8.04 (s, 1H), 7.99 (d, J=7.2 Hz, 1H), 7.88 (td, $J_{1}=7.7 \mathrm{~Hz}$, $\left.J_{2}=1.8 \mathrm{~Hz}, 2 \mathrm{H}\right), 7.77(\mathrm{~d}, J=8.0 \mathrm{~Hz}, 1 \mathrm{H}), 7.71-7.60(\mathrm{~m}, 4 \mathrm{H}), 7.53-7.43(\mathrm{~m}, 4 \mathrm{H})$, 7.36-7.33 (m, 2H).

Synthesis of Os(m-peptpy $)_{2}(\mathrm{TFSI})_{2}$.

$44 \mathrm{mg}$ (ca. $0.15 \mathrm{mmol})$ of $\mathrm{OsCl}_{3} \times \mathrm{xH}_{2} \mathrm{O}$ and $168 \mathrm{mg}(0.30 \mathrm{mmol})$ of $m$-peptpy were dispersed into $5 \mathrm{~mL}$ of ethylene glycol and refluxed for 2 hours at $230{ }^{\circ} \mathrm{C}$ with microwave radiation (Biotage Initiator 2.5). After cooling to room temperature, 433 $\mathrm{mg}$ of LiTFSI and $20 \mathrm{~mL}$ of DMF were added. The solution was stirred and water was added until black precipitates were produced. The precipitates were filtrated and washed with water. The solid was dispersed into hot toluene and collected by filtration. The solid was purified with acetonitrile and water to afford the solid of Os(m-peptpy $)_{2}(\mathrm{TFSI})_{2}$. (170 mg, 61\%) ${ }^{1} \mathrm{H}-\mathrm{NMR}\left(400 \mathrm{MHz}, \mathrm{DMSO}-d_{6}\right): \delta(\mathrm{ppm})$ $9.61(\mathrm{~s}, 4 \mathrm{H}), 9.13(\mathrm{~d}, J=8.0 \mathrm{~Hz}, 4 \mathrm{H}), 8.62(\mathrm{~s}, 2 \mathrm{H}), 8.58-8.55(\mathrm{~m}, 4 \mathrm{H}), 8.51-8.45(\mathrm{~m}$, $6 \mathrm{H}), 7.98-7.86(\mathrm{~m}, 10 \mathrm{H}), 7.81(\mathrm{~d}, J=8.4 \mathrm{~Hz}, 2 \mathrm{H}), 7.74(\mathrm{~d}, J=7.6 \mathrm{~Hz}, 2 \mathrm{H}), 7.70(\mathrm{~d}$, $J=7.6 \mathrm{~Hz}, 2 \mathrm{H}), 7.65-7.59(\mathrm{~m}, 6 \mathrm{H}), 7.46(\mathrm{~d}, J=5.6 \mathrm{~Hz}, 4 \mathrm{H}), 7.22$ (t, $J=6.6 \mathrm{~Hz}, 4 \mathrm{H})$ ${ }^{13} \mathrm{C}-\mathrm{NMR}\left(101 \mathrm{MHz}, \mathrm{DMSO}-d_{6}\right): \delta(\mathrm{ppm}) 159.7,154.6,152.1,145.6,141.0,139.0$, $137.6,135.7,134.3,132.4,131.6,130.9,130.4,130.4,130.2,129.3,128.9,128.3$, $128.1,127.8,127.8,127.3,127.1,127.0,125.4,124.9,121.0,120.8,120.4,119.8$, 
$119.4\left(J_{\mathrm{FC}}=323 \mathrm{~Hz}, \mathrm{CF}_{3}\right), \mathrm{MS}$ (MALDI): m/z $=1309.09\left(\left[\mathrm{Os}(m \text {-peptpy })_{2}-\mathrm{H}\right]^{+}\right)$, Elemental analysis: calculated for $\mathrm{C}_{86} \mathrm{H}_{50} \mathrm{~N}_{8} \mathrm{~F}_{12} \mathrm{O}_{8} \mathrm{OsS}_{4}: \mathrm{C} 55.24 \mathrm{H} 2.70 \mathrm{~N}$ 5.99, found C 55.04 H 2.66 N 5.99.

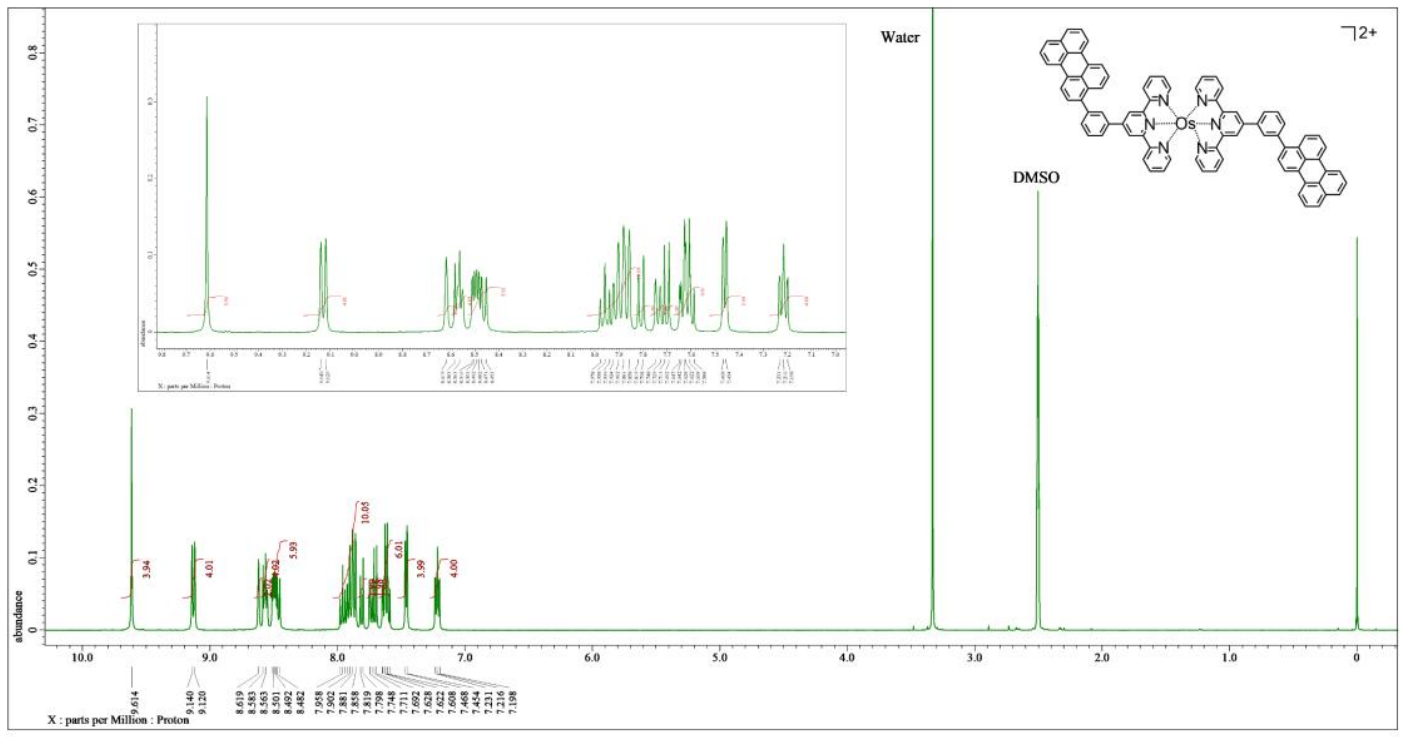

Figure S11. ${ }^{1} \mathrm{H}-\mathrm{NMR}$ spectrum of Os(m-peptpy $)_{2}(\mathrm{TFSI})_{2}\left(400 \mathrm{MHz}, \mathrm{DMSO}-d_{6}\right)$.

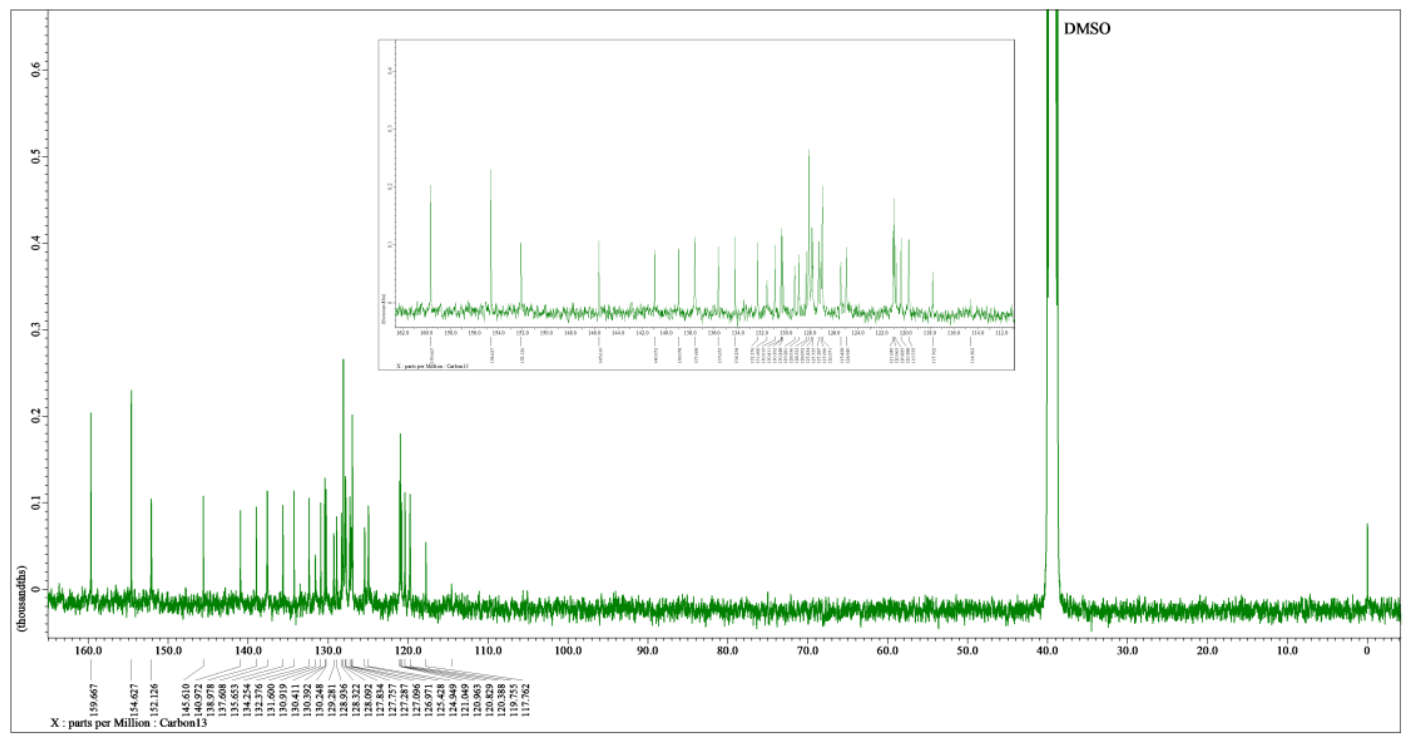

Figure S12. ${ }^{13} \mathrm{C}$-NMR spectrum of Os(m-peptpy $)_{2}(\mathrm{TFSI})_{2}\left(101 \mathrm{MHz}, \mathrm{DMSO}-d_{6}\right)$. A peak expected at 124.2 ppm for $\mathrm{CF}_{3}$ in $\mathrm{TFSI}^{-}$was not observed with sufficient resolution, but peaks at 121.0, 117.8, 114.6 were analyzed as a quartet. 

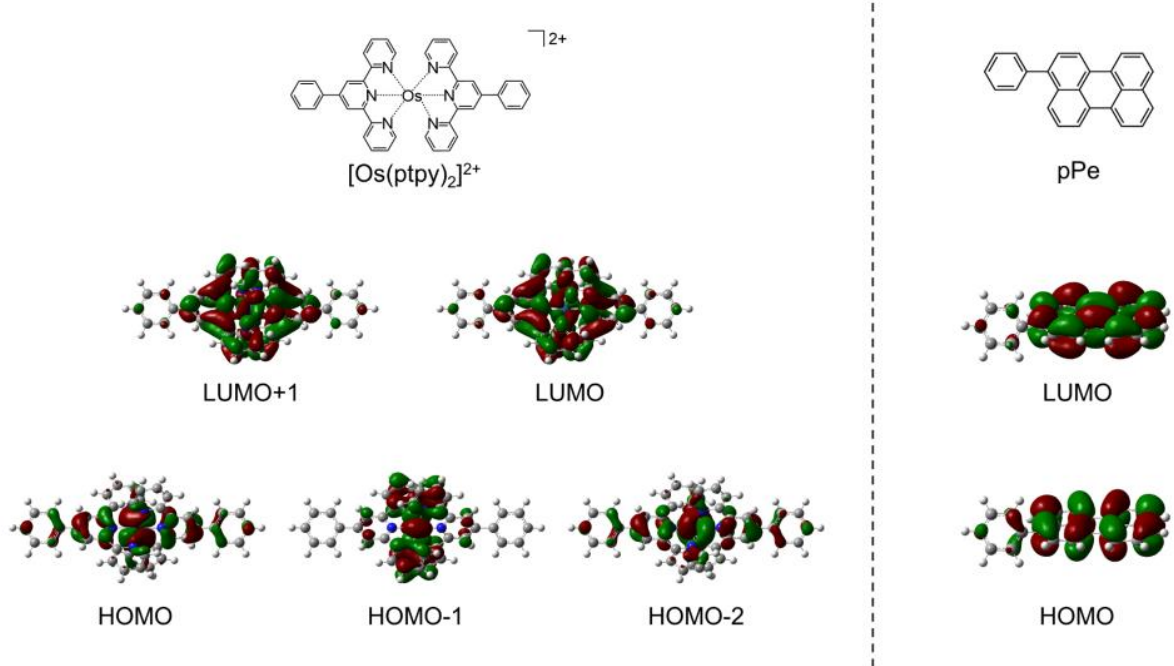

LUMO

glogese

НОМО

Figure S-13. Molecular orbitals of $\left[\mathrm{Os}(\mathrm{ptpy})_{2}\right]^{2+}$ and $\mathrm{pPe}$ in the ground $\left(\mathrm{S}_{0}\right)$ states (isovalue $=0.02$ ).

Table S-1 Calculated excited states of $\mathrm{pPe}$ with $\mathrm{S}_{0}$ optimized structure.

\begin{tabular}{|c|c|c|c|c|}
\hline $\begin{array}{c}\text { Excited } \\
\text { State }\end{array}$ & Transition & Coefficient & Energy (Wavelength) & $\begin{array}{c}\text { Oscillator } \\
\text { Strength }\end{array}$ \\
\hline \multirow{2}{*}{$\mathrm{T}_{1}$} & HOMO -> LUMO & 0.69731 & $1.5281 \mathrm{eV}(811.36 \mathrm{~nm})$ & 0.0000 \\
& HOMO <- LUMO & 0.12392 & & \\
\hline $\mathrm{S}_{1}$ & HOMO -> LUMO & 0.70576 & $2.6790 \mathrm{eV}(462.79 \mathrm{~nm})$ & 0.6199 \\
\hline
\end{tabular}


(a)

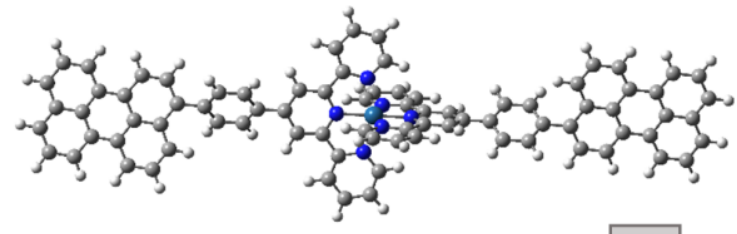

$\left[\mathrm{Os}(\text { peptpy })_{2}\right]^{2+} \quad \theta_{-1}$

$\mathrm{Pe}-\mathrm{ph}-\operatorname{tpy} \cdots$ Os $\cdots$ tpy $^{\prime}-\mathrm{ph}^{\prime}-\mathrm{Pe}^{\prime}$

\begin{tabular}{|c|c|c|c|c|}
\hline X1-X2 & Pe-ph & ph-tpy & tpy'-ph' & ph'-Pe' \\
\hline $\begin{array}{c}\theta(\mathrm{X} 1-X 2)^{\prime} \\
\text { in } \mathrm{S}_{0}\end{array}$ & $55.5^{\circ}$ & $-35.3^{\circ}$ & $35.0^{\circ}$ & $55.4^{\circ}$ \\
\hline $\begin{array}{c}\theta(\mathrm{X} 1-\mathrm{X} 2) \\
\text { in } \mathrm{T}_{1}\end{array}$ & $46.4^{\circ}$ & $-33.4^{\circ}$ & $36.2^{\circ}$ & $56.3^{\circ}$ \\
\hline
\end{tabular}

(c)

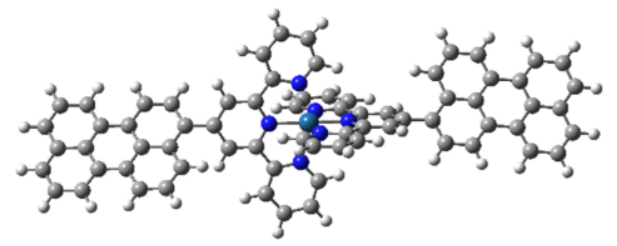

$\left[\mathrm{Os}(\text { petpy })_{2}\right]^{2+}$

$\mathrm{Pe}-$ tpy $\cdots$. Os $\cdots$...tpy' $-\mathrm{Pe}^{\prime}$

\begin{tabular}{|c|c|c|}
\hline $\mathrm{X} 1-\mathrm{X} 2$ & Pe-tpy & tpy'-Pe' $^{\prime}$ \\
\hline $\begin{array}{c}\theta(\mathrm{X} 1-\mathrm{X} 2) \\
\text { in } \mathrm{S}_{0}\end{array}$ & $54.2^{\circ}$ & $54.2^{\circ}$ \\
\hline $\begin{array}{c}\theta(\mathrm{X} 1-\mathrm{X} 2) \\
\text { in } \mathrm{T}_{1}\end{array}$ & $42.8^{\circ}$ & $54.7^{\circ}$ \\
\hline
\end{tabular}

Figure S-14. Optimized structures in $\mathrm{T}_{1}$ state and dihedral angle information of (a) $\left[\mathrm{Os}(\text { peptpy })_{2}\right]^{2+},(\mathrm{b})\left[\mathrm{Os}(m \text {-peptpy })_{2}\right]^{2+}$, and (c) $\left[\mathrm{Os}(\text { petpy })_{2}\right]^{2+}$ in $\mathrm{S}_{0}$ and $\mathrm{T}_{1}$ states.

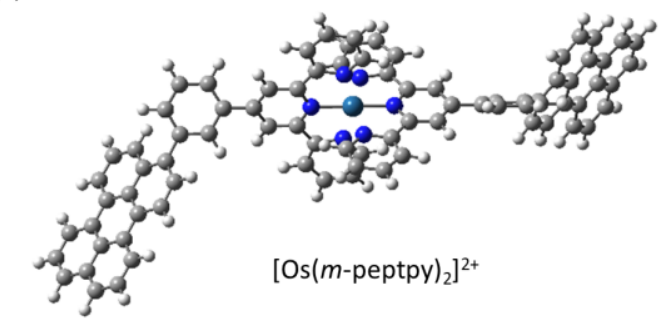

$\mathrm{Pe}-$ ph - tpy $\cdots$ Os $\cdots$ tpy - ph' $^{\prime}-\mathrm{Pe}^{\prime}$

\begin{tabular}{|c|c|c|c|c|}
\hline $\mathrm{X} 1-\mathrm{X} 2$ & Pe-ph & ph-tpy & tpy'-ph' & ph'-Pe' \\
\hline $\begin{array}{c}\theta(\mathrm{X} 1-\mathrm{X} 2) \\
\text { in } \mathrm{S}_{0}\end{array}$ & $58.2^{\circ}$ & $37.0^{\circ}$ & $-36.9^{\circ}$ & $-59.0^{\circ}$ \\
\hline $\begin{array}{c}\theta(\mathrm{X} 1-\mathrm{X} 2) \\
\text { in } \mathrm{T}_{1}\end{array}$ & $51.5^{\circ}$ & $37.2^{\circ}$ & $-37.2^{\circ}$ & $-59.0^{\circ}$ \\
\hline
\end{tabular}


(a)

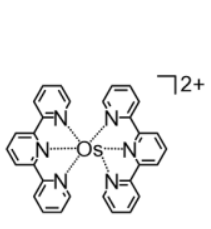

$\left[\mathrm{Os}(\mathrm{tpy})_{2}\right]^{2+}$ (b)

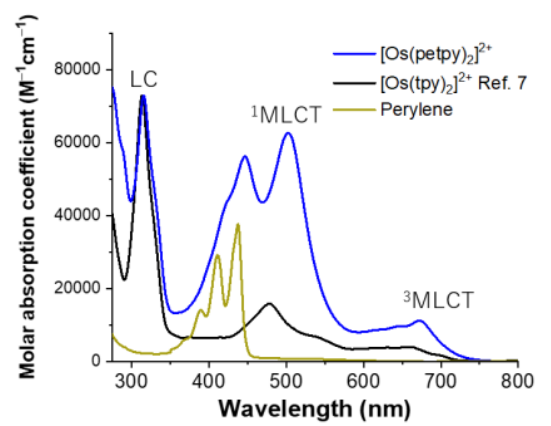

(c)

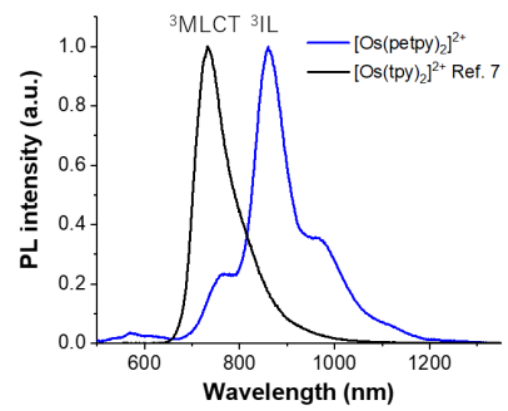

Figure S-15. (a) Chemical structure of $\left[\mathrm{Os}(\mathrm{tpy})_{2}\right]^{2+}$. (b) Absorption spectra of $\left[\mathrm{Os}(\text { petpy) })_{2}\right]^{2+}$ (blue), $\left[\mathrm{Os}(\text { tpy) })_{2}\right]^{2+}$ (gray) and perylene (dark yellow) in DMF. (c) Emission spectra of $\left[\text { Os(petpy) }{ }_{2}\right]^{2+}$ (blue, $20 \mu \mathrm{M}, \lambda_{\text {ex }}=350 \mathrm{~nm}$ ), $\left[\text { Os(petpy) }{ }_{2}\right]^{2+}$ (gray, 20 $\left.\mu \mathrm{M}, \lambda_{\mathrm{ex}}=480 \mathrm{~nm}\right)$ in DMF. Spectra of $\left[\mathrm{Os}(\mathrm{tpy})_{2}\right]^{2+}$ were reproduced in part with permission from from Ref. 7. Copyright 2020, the Royal Society of Chemistry.

(a)

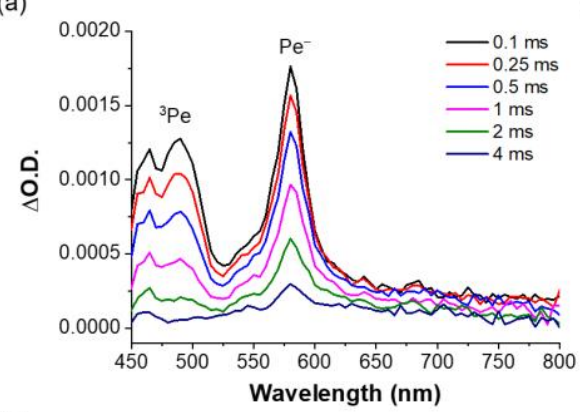

(c)

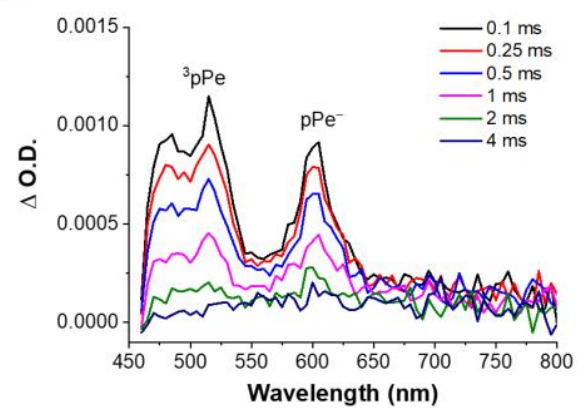

(b)

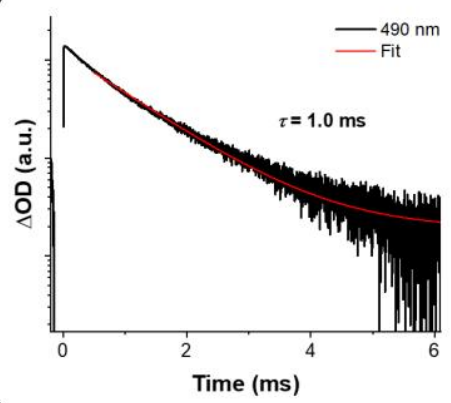

(d)

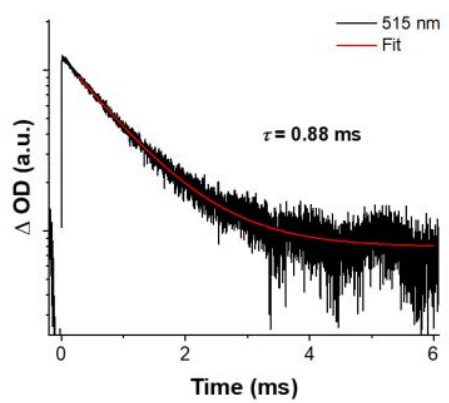

Figure S-16. Transient absorption spectra and the transient signal decays of $(a, b)$ perylene $\left(\lambda_{\mathrm{ex}}=437 \mathrm{~nm}\right)$ and $(\mathrm{c}, \mathrm{d}) 3$-phenylperylene $\left(\lambda_{\mathrm{ex}}=447 \mathrm{~nm}\right)$. The peaks corresponding with triplet-to-triplet absorption of perylene at $490 \mathrm{~nm}^{8}$ and 3phenylperylene at $515 \mathrm{~nm}$ were fitted by a single exponential decay function. 
(a)

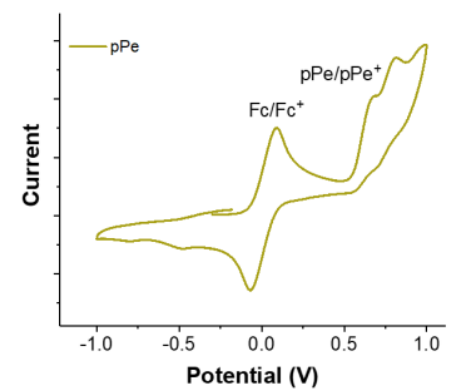

(c)

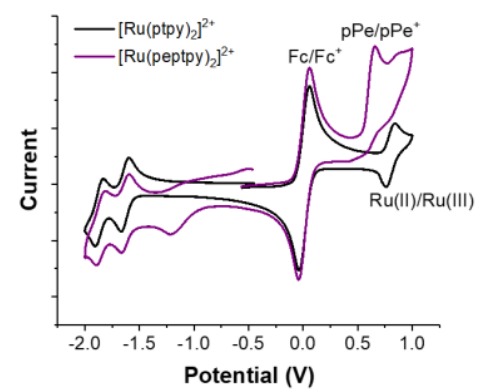

(b)

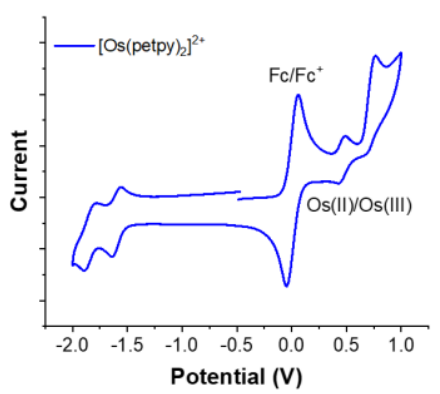

Figure S-17. Cyclic voltammograms for $1 \mathrm{mM}$ (a) pPe, (b) $\left[\mathrm{Os}(\text { petpy })_{2}\right]^{2+}$, (c) $\left[R u(p t p y)_{2}\right]^{2+}$ and $\left[R u(\text { peptpy })_{2}\right]^{2+}$ in DMF in the presence of $0.1 \mathrm{M}$ LiTFSI (working electrode: glassy carbon, counter electrode: $\mathrm{Pt}$, reference electrode: $\mathrm{Ag} / \mathrm{AgCl}$, Sweep: 0.5 $\mathrm{V} / \mathrm{s}$ for $\mathrm{pPe}$ and $0.1 \mathrm{~V} / \mathrm{s}$ for others, internal standard: $2 \mathrm{mM}$ Ferrocene). 


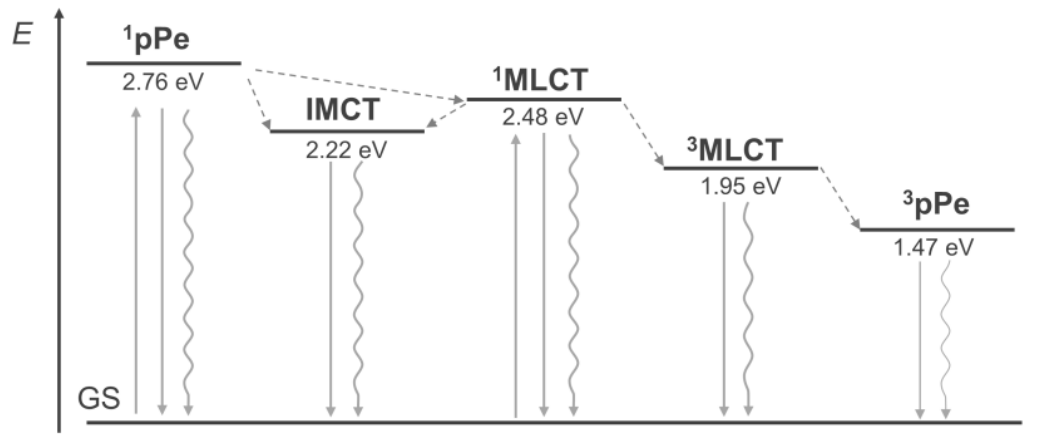

Figure S-18. Energy levels of excited singlet and triplet states of $\left[\mathrm{Ru}(\text { peptpy })_{2}\right]^{2+}$. Note that ${ }^{1} \mathrm{pPe}$ and ${ }^{1} \mathrm{MLCT}$ states were determined by the absorption peaks. IMCT state was estimated by cyclic voltammetry.

\section{References}

1. Sasaki, Y.; Oshikawa, M.; Bharmoria, P.; Kouno, H.; Hayashi - Takagi, A.; Sato, M.; Ajioka, I.; Yanai, N.; Kimizuka, N. Near-Infrared Optogenetic Genome Engineering Based on Photon-Upconversion Hydrogels. Angew. Chem., Int. Ed. 2019, 58, 17827-17833.

2. Frisch, M. J.; Trucks, G. W.; Schlegel, H. B.; Scuseria, G. E.; Robb, M. A.; Cheeseman, J. R.; Scalmani, G.; Barone, V.; Petersson, G. A.; Nakatsuji, H.; Li, X.; Caricato, M.; Marenich, A. V.; Bloino, J.; Janesko, B. G.; Gomperts, R.; Mennucci, B.; Hratchian, H. P.; Ortiz, J. V.; Izmaylov, A. F.; Sonnenberg, J. L.; Williams-Young, D.; Ding, F.; Lipparini, F.; Egidi, F.; Goings, J.; Peng, B.; Petrone, A.; Henderson, T.; Ranasinghe, D.; Zakrzewski, V. G.; Gao, J.; Rega, N.; Zheng, G.; Liang, W.; Hada, M.; Ehara, M.; Toyota, K.; Fukuda, R.; Hasegawa, J.; Ishida, M.; Nakajima, T.; Honda, Y.; Kitao, O.; Nakai, H.; Vreven, T.; Throssell, K.; Montgomery Jr., J. A.; Peralta, J. E.; Ogliaro, F.; Bearpark, M. J.; Heyd, J. J.; Brothers, E. N.; Kudin, K. N.; Staroverov, V. N.; Keith, T. A.; Kobayashi, R.; Normand, J.; Raghavachari, K.; Rendell, A. P.; Burant, J. C.; Iyengar, S. S.; Tomasi, J.; Cossi, M.; Millam, J. M.; Klene, M.; Adamo, C.; Cammi, R.; Ochterski, J. W.; Martin, R. L.; Morokuma, K.; Farkas, O.; Foresman, J. B.; Fox, D. J. Gaussian 16, Revision B.01, Gaussian, Inc., Wallingford CT 2016.

3. Dennington, R.; Keith, T. A.; Millam, J. M. GaussView, Version 6, Semichem Inc., Shawnee Mission, KS, 2016.

4. Cargill Thompson, A. M. W. The synthesis of 2,2':6',2"-terpyridine ligands - versatile building blocks for supramolecular chemistry. Coord. Chem. Rev. 1997, 160, 1-52.

5. Jarosz, P.; Du, P.; Schneider, J.; Lee, S.-H.; McCamant, D.; Eisenberg, R. Platinum(II) Terpyridyl Acetylide Complexes on Platinized $\mathrm{TiO}_{2}$ : Toward the Photogeneration of $\mathrm{H}_{2}$ in 
Aqueous Media. Inorg. Chem. 2009, 48, 9653-9663.

6. Collin, J. P.; Guillerez, S.; Sauvage, J. P. Ruthenium and osmium complexes of 2, 2': 6', 2 "-terpyridine covalently linked to electron acceptor and electron donor groups. J. Chem. Soc., Chem. Commun. 1989, 776-778.

7. Haruki, R.; Sasaki, Y.; Masutani, K.; Yanai, N.; Kimizuka, N. Leaping across the visible range: near-infrared-to-violet photon upconversion employing a silyl-substituted anthracene. Chem. Commun. 2020, 56, 7017-7020.

8. Konuk, R.; Cornelisse, J.; McGlynn, S. P. Photoionization of perylene in polar solvents. J. Chem. Phys. 1985, 82, 3929-3933. 